\title{
Active DNA demethylation of developmental cis-regulatory regions predates vertebrate origins
}

Ksenia Skvortsova ${ }^{1,2 *}$, Stephanie Bertrand ${ }^{3}$, Danila Voronov ${ }^{4}$, Paul E. Duckett ${ }^{1}$, Samuel E. Ross ${ }^{1,2}$, Marta Silvia Magri ${ }^{5}$, Ignacio Maeso ${ }^{5}$, Robert J. Weatheritt ${ }^{1,6}$, Jose Luis Gómez Skarmeta ${ }^{5, \#}$, Maria Ina Arnone ${ }^{4}$, Hector Escriva ${ }^{3}$, Ozren Bogdanovic ${ }^{1,7^{*}}$

${ }^{1}$ Genomics and Epigenetics Division, Garvan Institute of Medical Research, Sydney, Australia

${ }^{2}$ St Vincent's Clinical School, Faculty of Medicine, UNSW, Sydney, Australia

${ }^{3}$ Sorbonne Université, CNRS, Biologie Intégrative des Organismes Marins (BIOM), Observatoire Océanologique, Banyuls-sur-

Mer, France

${ }^{4}$ Biology and Evolution of Marine Organisms (BEOM), Stazione Zoologica Anton Dohrn, Naples, Italy

${ }^{5}$ Centro Andaluz de Biología del Desarrollo, CSIC-Universidad Pablo de Olavide-Junta de Andalucía, Seville, Spain

${ }^{6}$ EMBL Australia, Garvan Institute of Medical Research, Sydney, Australia.

${ }^{7}$ School of Biotechnology and Biomolecular Sciences, University of New South Wales, Sydney, Australia

* Correspondence to: k.skvortsova@garvan.org.au and o.bogdanovic@ garvan.org.au

\# Deceased

\begin{abstract}
DNA methylation (5-methylcytosine; $5 \mathrm{mC}$ ) is a repressive gene-regulatory mark required for vertebrate embryogenesis. Genomic $5 \mathrm{mC}$ is tightly regulated through the coordinated action of DNA methyltransferases, which deposit $5 \mathrm{mC}$, and TET enzymes, which participate in its active removal through the formation of 5hydroxymethylcytosine $(5 \mathrm{hmC})$. TET enzymes are essential for mammalian gastrulation and activation of vertebrate developmental enhancers, however, to date, a clear picture of $5 \mathrm{hmC}$ function, abundance, and genomic distribution in non-vertebrate lineages is lacking. By employing base-resolution $5 \mathrm{mC}$ and $5 \mathrm{hmC}$ quantification during sea urchin and lancelet embryogenesis, we shed light on the roles of non-vertebrate $5 \mathrm{hmC}$ and TET enzymes. We find that these invertebrate deuterostomes employ TET enzymes for targeted demethylation of regulatory regions associated with developmental genes and show that the complement of identified $5 \mathrm{hmC}$-regulated genes is conserved to vertebrates. This work thus demonstrates that active $5 \mathrm{mC}$ removal from regulatory regions is a common feature of deuterostome embryogenesis suggestive of unexpected deep conservation of a major gene-regulatory module.
\end{abstract}

Keywords: DNA methylation, embryogenesis, evolution, TET enzymes, 5hmC

\section{Introduction}

Metazoan development is governed by the activity of cis-regulatory DNA elements that serve as binding platforms for transcription factors controlling spatial and temporal gene expression ${ }^{1-4}$. In vertebrates, one of the major gene-regulatory mechanisms entails methylation of the $5^{\text {th }}$ carbon of the cytosine pyrimidine ring $(5 \mathrm{mC})$ within the $\mathrm{DNA}^{5} .5 \mathrm{mC}$ is a predominantly repressive gene-regulatory mark, which in vertebrates displays unparalleled developmental dynamics. Mammalian embryogenesis is characterised by two rounds of genome-wide $5 \mathrm{mC}$ reprogramming that take place in the preimplantation embryo and in the developing germline ${ }^{6,7}$. Global $5 \mathrm{mC}$ erasure occurs through a combination of active and passive $5 \mathrm{mC}$ removal mechanisms, such as the exclusion of maintenance DNA methyltransferase (DNMT1) from the nucleus ${ }^{8-12}$, and targeted oxidation of $5 \mathrm{mC}$ by Ten Eleven Translocation (TET) enzymes, respectively ${ }^{13-15}$. TET enzymes oxidise $5 \mathrm{mC}$ through a series of reactions involving 5-hydroxymethylcytosine ( $5 \mathrm{hmC}), 5$-formylcytosine (5fC), and 5-carboxylcytosine (5caC) intermediates, the latter two of which can be excised by base excision repair (BER) pathways, thus leading to the restoration of unmethylated cytosine at affected sites $^{16,17}$. Apart from participating in mammalian global $5 \mathrm{mC}$ erasure, TET enzymes drive targeted $5 \mathrm{mC}$ removal from promoters and enhancers required for vertebrate gastrulation ${ }^{18}$ and organ formation ${ }^{19}$. TET activity is thus crucial for orchestrating gene regulatory programs associated 
with cell fate determination and tissue differentiation. TET depletion accompanied by $5 \mathrm{hmC}$ reduction results in enhancer hypermethylation and reduced chromatin accessibility in vertebrate embryos ${ }^{19,20}$, delayed gene induction during cellular differentiation $^{21}$, and skewed lineage differentiation $^{22-24}$. Loss of $5 \mathrm{mC}$ in DNMT mutant cells, on the other hand, was shown to result in the activation of intragenic enhancers ${ }^{25}$, altogether highlighting the significance of timely DNA demethylation in the regulation of cis-regulatory elements and lineage specification.

In stark contrast to vertebrates, which are characterized by high genomic $5 \mathrm{mC}$ levels ( 80\%), most nonvertebrate organisms sampled to date possess mosaic DNA methylation patterns that vary widely in their content, genomic distribution, and function ${ }^{26-28}$. Notable exceptions to that rule include Drosophila melanogaster $^{29}$ and Caenorhabditis elegans ${ }^{30}$ whose genomes are devoid of $5 \mathrm{mC}$, and the demosponge Amphimedon queenslandica, the genome of which displays vertebrate-like genomic hypermethylation $^{31}$. Those exceptions notwithstanding, a common feature of invertebrate $5 \mathrm{mC}$ patterning is the association of $5 \mathrm{mC}$ with gene bodies of actively transcribed genes ${ }^{26} .5 \mathrm{mC}$ has been shown to prevent precocious transcription from cryptic transcription start sites located within gene bodies ${ }^{32}$. However, it is currently underexplored whether any other aspects of 5mC-mediated genome regulation are evolutionarily maintained across the invertebrate-vertebrate boundary. For example, TET proteins are present in most non-vertebrate genomes ${ }^{33}$ where their function has been linked to RNA hydroxymethylation ${ }^{34}$, production of $5 \mathrm{hmC}$ in the germline ${ }^{35}$, or regulation of alternative splicing ${ }^{36}$. Except for a single study that employed ELISA-based approaches to demonstrate global $5 \mathrm{hmC}$ developmental loss in the Pacific sea gooseberry (Pleurobrachia bachei) ${ }^{37}$, it is not yet clear to what extent non-vertebrate organisms utilise $5 \mathrm{mC}$ and consequently TET proteins and $5 \mathrm{hmC}$ for developmental gene regulation. Moreover, base-resolution hydroxymethylomes have thus far only been generated for sponges and sea anemones (discussed below) ${ }^{31}$, leaving numerous open questions as to the quantity, genomic localisation, and function of non-vertebrate $5 \mathrm{hmC}$.
While it is well-established that non-vertebrate genomes exhibit low $5 \mathrm{mC}$, it is worth noting that cisregulatory elements frequently reside within highly methylated gene bodies. It is thus possible that the $5 \mathrm{mC}$ state of such elements is regulated independenttly of bulk gene body $5 \mathrm{mC}$. For example, we have recently shown that the European lancelet (Branchiostoma lanceolatum), an invertebrate chordate, exhibits developmental DNA demethylation of cisregulatory elements located within methylated gene bodies $^{38}$. Notably, this demethylation event coincided with the expression of the lancelet TET orthologue, thereby suggesting that animals with mosaic $5 \mathrm{mC}$ might employ TET-mediated demethylation of enhancers for their spatio-temporal regulation during development. $5 \mathrm{hmC}$ profiling of genomic DNA extracted from the demosponge $A$. queenslandica demonstrated enrichment of this mark at potential transcription factor binding sites ${ }^{31}$. Nevertheless, definitive proof that TET proteins and active DNA demethylation participate in developmental enhancer or promoter usage in nonvertebrate lineages, has not been provided to date. To better understand the evolution of TET-mediated regulation of distal regulatory elements, we conducted detailed analyses of genome-wide $5 \mathrm{mC}$ and $5 \mathrm{hmC}$ dynamics during development of the purple sea urchin (Strongylocentrotus purpuratus), which forms part of the sister lineage to chordates, the ambulacrarians ${ }^{39}$, and the invertebrate chordate European lancelet ${ }^{38}$. Specifically, we employed whole genome bisulfite sequencing (WGBS) ${ }^{40}$ and APOBEC-coupled epigenetic sequencing (ACEseq $)^{41}$ to generate base-resolution maps of $5 \mathrm{mC}$ and $5 \mathrm{hmC}$, respectively, at four developmental time points. Integration of these datasets with open chromatin profiles demonstrated prominent active DNA demethylation of regulatory regions in both examined organisms, thus strongly supporting the notion that invertebrate deuterostomes employ TET proteins and $5 \mathrm{hmC}$ for developmental gene regulation. Moreover, we found that vertebrate orthologues of genes marked by $5 \mathrm{hmC}$ in these invertebrate deuterostomes are associated with $5 \mathrm{hmC}$ enrichment and defined spatio-temporal regulatory logic in zebrafish. Altogether, our work demonstrates that at least in some non-vertebrate lineages, $5 \mathrm{mC}$ is not merely a by-product of geneactivity required for the prevention of spurious transcriptional initiation, but a bona-fide regulatory 
mark that is being actively remodelled at regulatory regions associated with key developmental processes.

\section{Results \\ Structural and functional conservation of TET enzymes}

To better understand the evolutionary conservation of 5hmC and TET expression dynamics during embryogenesis, we first wanted to determine whether echinoderm and cephalochordate TET enzymes are characterised by the same protein domain composition as vertebrate TETs. The sea urchin genome ${ }^{39}$ as well as the genomes of the European $^{38}$ and Florida lancelet ${ }^{42}$ encode a single copy of TET proteins. The genomes of most vertebrates, however, exhibit two or three TET copies $^{43,44}$ (Fig. S1A), which arose during the process of whole genome duplication ${ }^{45}$. Comparison of TET protein structure revealed that both the sea urchin TET (sTET) as well as the lancelet TET (bTET) contain an $\mathrm{N}$-terminal $\mathrm{CxxC}$ domain, which is also present in vertebrate TET1 and TET3 enzymes (Fig. 1A $)^{46}$. CxxC domains can interact directly with the DNA thereby facilitating TET interaction with chromatin ${ }^{47,48}$. The C-terminus of sTET and bTET is characterised by a doublestranded $\beta$-helix (DSBH) domain that harbours binding sites for the $\mathrm{Fe}$ (II) and 2-oxoglutarate (2OG) cofactors required for TET enzymatic activity ${ }^{46}$. Notably, the DSBH domain in sTET, bTET and vertebrate TET proteins is interrupted by a sizable low complexity region (Fig. 1A). These results are in line with the previously described structural conservation of metazoan TET proteins ${ }^{33}$. We next conducted multiple sequence alignment analyses and found strong sequence conservation in the core catalytic domain (Fig. 1B, Fig. S1B), as well as striking structural conservation between sTET, bTET and their vertebrate counterparts (Fig. 1C, Fig. S1C-E). Having determined the suitability of sea urchin and lancelet for TET evolutionary comparisons and $5 \mathrm{hmC}$ profiling, we next quantified TET expression during sea urchin, lancelet, and zebrafish development ${ }^{38,49}$. In all examined species, the developmental peak of TET expression could be observed during mid-development (Fig. 1D), coinciding with gastrulation (sea urchin) and segmentation (lancelet, zebrafish) (Fig. S1F) ${ }^{50,51}$. These observations are in accord with reduced developmental requirements for TET activity during vertebrate pluripotency ${ }^{18-20,22}$. Overall, our results demonstrate highly conserved TET protein structure and developmental TET expression profiles across diverse deuterostomes, suggestive of the important roles these proteins might play during animal development.

\section{DNA methylation content and dynamics of sea urchin and lancelet genomes}

To quantify the amount of genomic $5 \mathrm{mC}$, which can act as substrate for TET oxidation and $5 \mathrm{hmC}$ formation, we generated base-resolution $5 \mathrm{mC}$ profiles (WGBS $)^{40}$ corresponding to the following stages of sea urchin development: 24hpf (blastula), 48hpf (gastrula), 72hpf (pluteus), adult (tube feet). Additionally, we analysed WGBS datasets of lancelet embryogenesis from 8hpf (blastula - G3), 15hpf (gastrula - G6), 36hpf (neurula - T0), and adult (liver - A) time points (Fig. 2A, Table S1) ${ }^{38,50,51}$. In agreement with previous studies ${ }^{38,52}$, average methylation values (sea urchin $=23-28 \%$, lancelet $=$ 21-25\%) (Fig. 2A) were consistent with canonical invertebrate methylome patterns, where the majority of genomic $5 \mathrm{mC}$ is located within gene bodies of expressed genes (Fig. S2A-C) ${ }^{28}$. Notably, the adult samples of both the sea urchin and the lancelet displayed considerably lower $5 \mathrm{mC}$ levels reminiscent of developmental $5 \mathrm{mC}$ dynamics in zebrafish and Xenopus tropicalis (Fig. 2A) ${ }^{19}$. Given the positive correlation between gene expression and gene body $5 \mathrm{mC}$ in non-vertebrate organisms, lower $5 \mathrm{mC}$ levels in adult tissues could potentially be explained by specialised transcriptional profiles of those tissues characterised by a smaller number of expressed genes or a lower transcriptional output overall. To that end, we analysed the transcripttomes ${ }^{38,49}$ of multiple developmental stages and adult tissues and revealed comparable mean expression profiles across all examined samples in both organisms (Fig. 2B). These results suggest that the observed loss of $5 \mathrm{mC}$ in adults is likely not caused by major changes in transcriptional complexity but is rather linked to a gradual increase in chromatin accessibility associated with more frequent usage of cis-regulatory elements ${ }^{53}$. To obtain further insight into developmental dynamics of $5 \mathrm{mC}$, we identified genomic regions, which display significant $(F D R<$ $0.05, \Delta \mathrm{mCG} / \mathrm{CG} \geq 0.2$ ) developmental changes in 5mC levels (Fig. 2C, Table S2-S7). 
A

Strongylocentrotus purpuratus

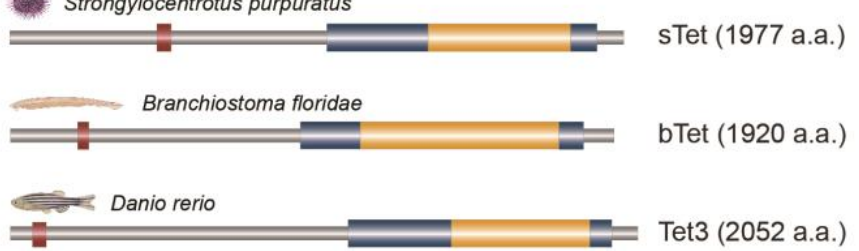

- $\mathrm{CxxC}$ low complexity region $=\mathrm{DSBH}$ (dioxygenase activity)

B

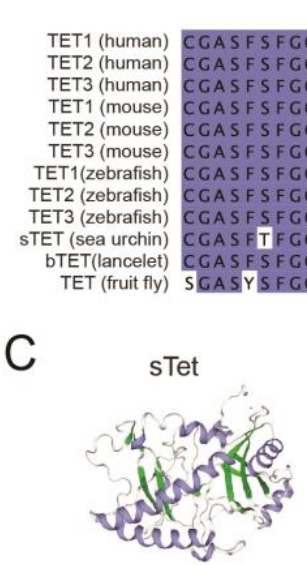
Interaction
with DNA
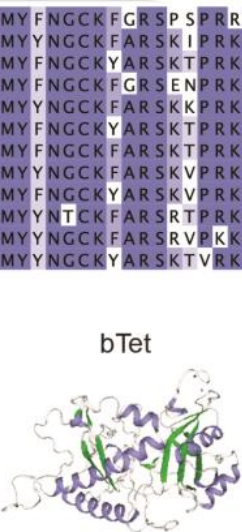

\section{2-OG Substrate} binding binding
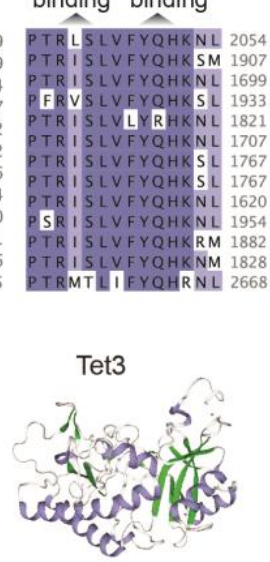
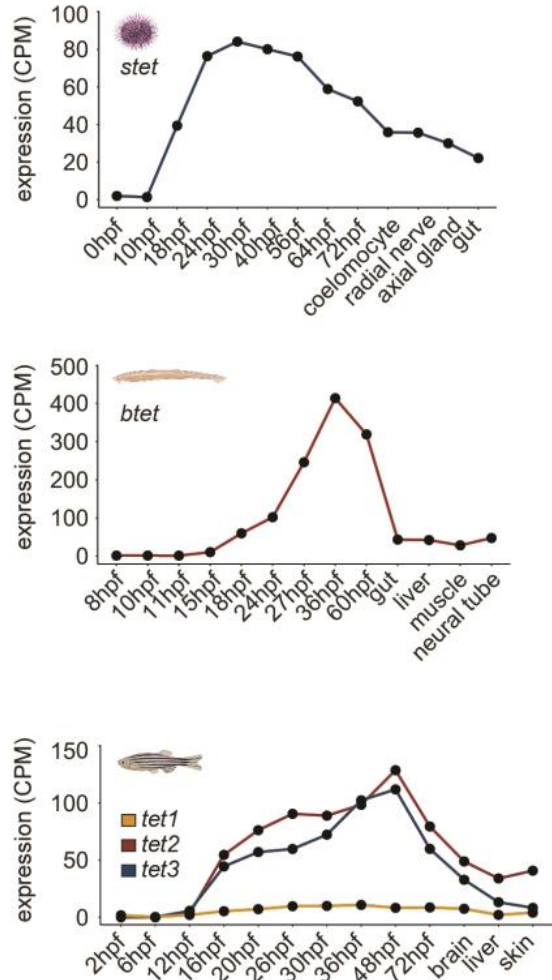

Figure 1. Evolutionary conservation of TET protein sequence, catalytic domain structure, and developmental expression. (A) Sea urchin, lancelet and zebrafish TET protein domain structure predicted using SWISS-MODEL and HMMER. Sea urchin sTet, Florida lancelet bTet, and zebrafish Tet3 harbour an N-terminal DNA-binding CxxC domain and a C-terminal catalytic double stranded $\beta$-helix domain (DSBH) containing a large low-complexity insert. (B) Multiple sequence alignment of human, mouse, and zebrafish TET1, TET2, TET3 as well as sea urchin, lancelet, and fruit fly TET core catalytic domain. A part of DSBH is shown. The colour of each amino acid indicates percentage identity (PID) with darker blue depicting higher PID and lighter blue - lower PID. (C) 3D models of methylcytosine dioxygenase domains of sea urchin sTet, lancelet bTet, and zebrafish Tet3 performed using SWISS-MODEL. Crystal structure of the human TET2-5fC complex (5d9y.1.A) was ranked as a top template in the template search and was used for model building. a-helix is depicted in blue and $\beta$-sheet - in green. (D) TET gene expression dynamics during sea urchin, lancelet, and zebrafish development.

We identified 20,072 and 8,136 of such differentially methylated regions (DMRs) in sea urchin and lancelet genomes, respectively, with the majority corresponding to the larva/pluteus-to-adult transitions (Fig. 2D, Fig. S2D). The majority $(86 \%$ sea urchin, $80 \%$ lancelet) of the identified DMRs were associated with developmental hypomethylation (Fig. 2C-D, Fig. S2D). These results are in agreement with the overall lower levels of $5 \mathrm{mC}$ in adult tissues in both organisms (Fig. 2A). Altogether, we find that the embryogenesis of sampled invertebrate deuterostomes is characterised by significant developmental loss of $5 \mathrm{mC}$, which cannot be explained by developmental changes in transcriptional activity.

\section{Developmental dynamics of the 5-hydroxymethy- Icytosine landscape}

To explore the developmental dynamics, genomic content, and distribution of $5 \mathrm{hmC}$ in sea urchin and lancelet genomes and to compare those against vertebrate $5 \mathrm{hmC}$ patterns, we employed nondestructive, base-resolution sequencing of $5 \mathrm{hmC}$ using a DNA deaminase (ACE-seq) ${ }^{41}$. We first wanted to test how ACE-seq $5 \mathrm{hmC}$ quantification compares to TET-assisted bisulfite sequencing $(\mathrm{TAB}-\mathrm{seq})^{54}$ and immunoprecipitation (hMeDIPseq) ${ }^{55} 5 \mathrm{hmC}$ profiles in zebrafish, an organism with a well-described DNA hydroxymethylome ${ }^{19,22,56,57}$. Firstly, we found that the same genomic regions displayed $5 \mathrm{hmC}$ enrichment identified by all the three techniques (Fig. S3A, B). Furthermore, we observed a significant overlap between data originating from the two base-resolution approaches 
A

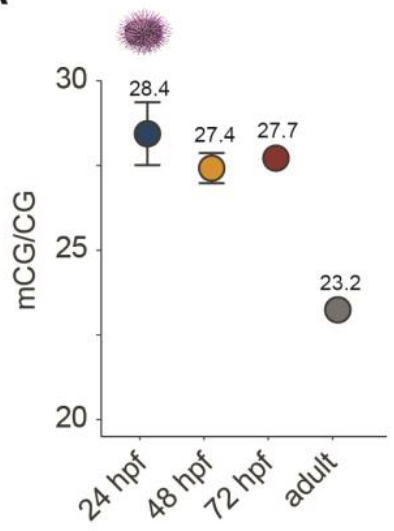

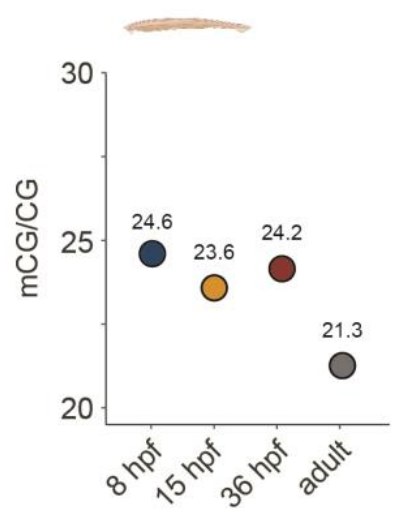
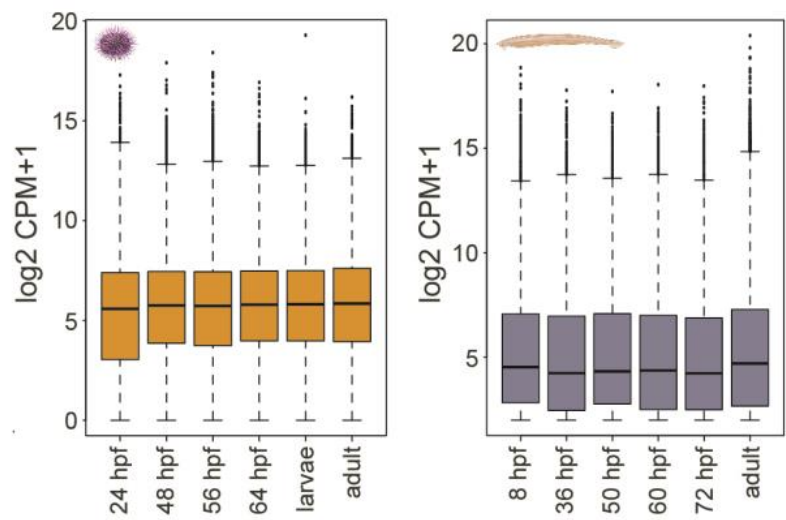

C
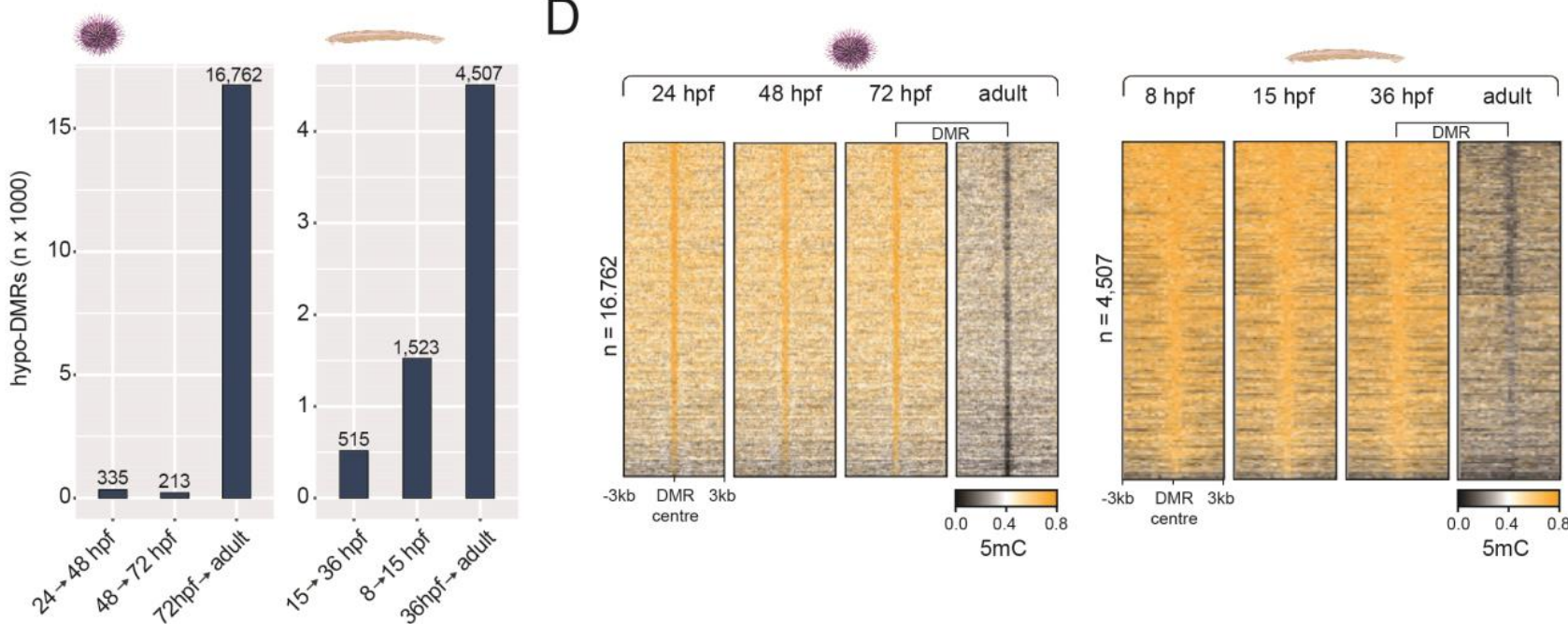

Figure 2. DNA methylation dynamics of sea urchin and lancelet development. (A) Average DNA methylation (mCG/CG) levels during sea urchin and lancelet development. (B) Average gene expression $(C P M+1)$ levels during sea urchin and lancelet development. (C) Number of hypomethylated DMRs (hypoDMRs) identified between embryonic and adult stages in sea urchin and lancelet genomes. (D) Heatmaps depicting DNA methylation (mCG/CG) at adult hypoDMRs in sea urchin and lancelet genomes.

(Fig. S3C) and hence proceeded to generate sea urchin and lancelet 5hmC maps using ACE-seq. We obtained DNA hydroxymethylome profiles for the following stages of sea urchin and lancelet embryogenesis: 24hpf (blastula), 48hpf (gastrula), 72hpf (pluteus), adult (tube feet), and 36hpf (late neurula - T0), 60-72hpf (larvae - L0), 3-4mo (juvenile - J), adult (liver - A) $)^{50,51}$ (Table S1). Identification of hydroxymethylated sites (proportion test, $P$ adj. $<0.05, \Delta$ hmCG/CG $\geq 0.1)^{58}$ in sea urchin and lancelet genomes, revealed $1-2 \%$ of CpGs with significant $5 \mathrm{hmC}$ content (Fig. 3A). In zebrafish $24 \mathrm{hpf}$ embryos, which are characterised by strong TET activity (Fig. 1D), 5\% of overall CpGs were identified as significantly hydroxymethylated (Fig. 3A). These results are in line with the overall higher global $5 \mathrm{mC}$ content of vertebrate genomes (Fig. 3A). To assess the reproducibility of ACE-seq
5hmC data between replicates, we searched the genome for sites of significant $5 \mathrm{hmC}$ enrichment and plotted mean $5 \mathrm{hmC}$ values between replicates (Fig. 3B). We observed an overall positive correlation of $5 \mathrm{hmC}$ levels in those regions with correlation values of $R=0.58$ and $R=0.68$ for sea urchin and lancelet, respectively. Given the transient nature of $5 \mathrm{hmC}$ and its role in $5 \mathrm{mC}$ to $\mathrm{C}$ conversion, the expectation would be for the majority of $5 \mathrm{hmC}$ sites to exist in a fully methylated $(5 \mathrm{mC})$ state before the onset of TET activity and in a fully demethylated (C) state once the period of TET-mediated demethylation has ended. To test whether this holds true in amphioxus and sea urchin genomes, we identified significant $5 \mathrm{hmC}$ sites at the stage coinciding with high TET expression (48hpf - sea urchin; 60hpf lancelet) and interrogated their fate before and after this period (Fig. 3C, D). Expectedly, we found that the majority of hydroxy- 
A

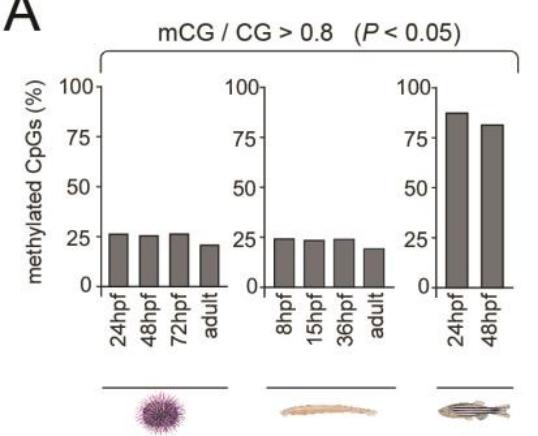

C

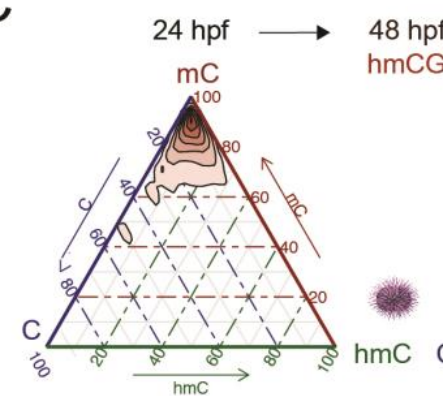

E

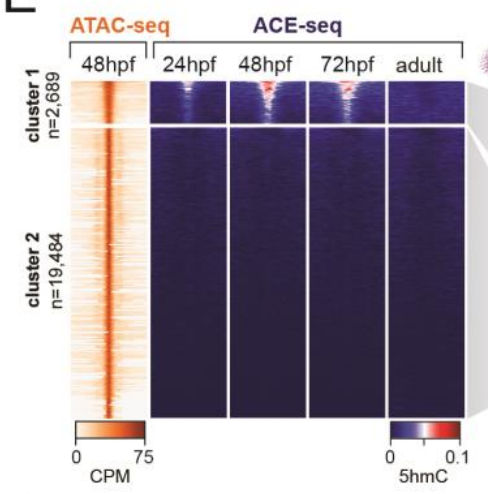

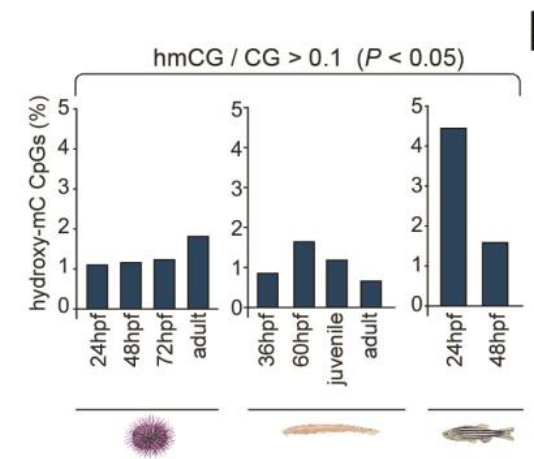

B

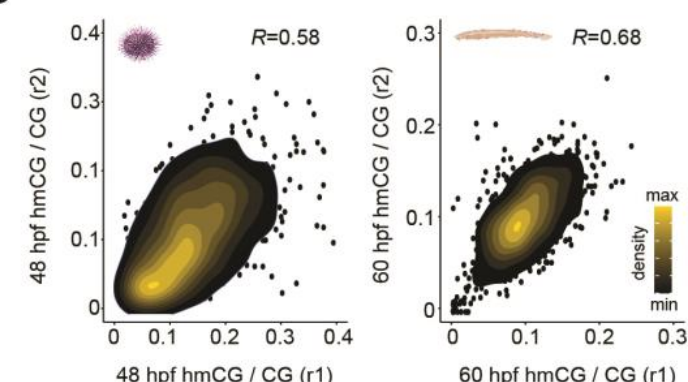

G
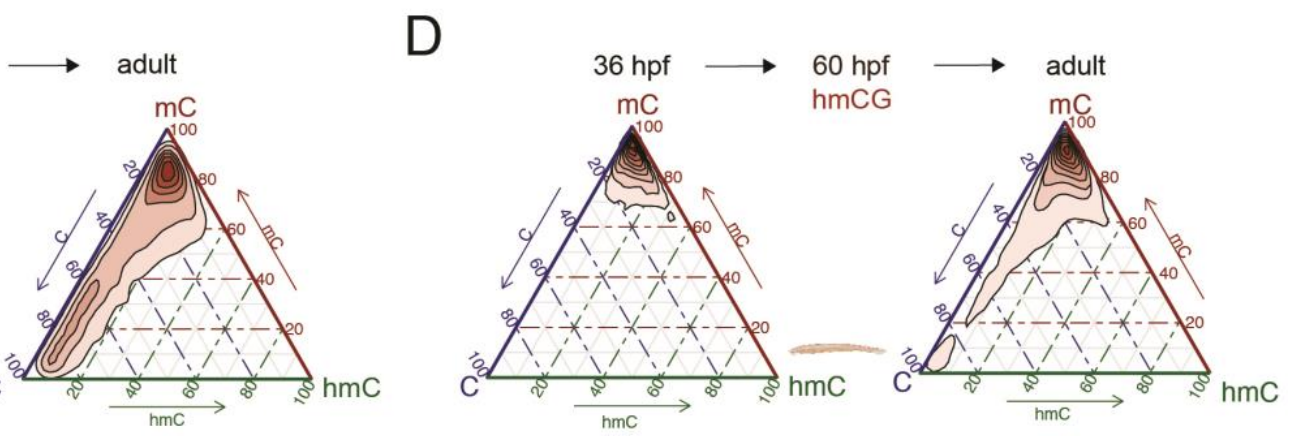

F
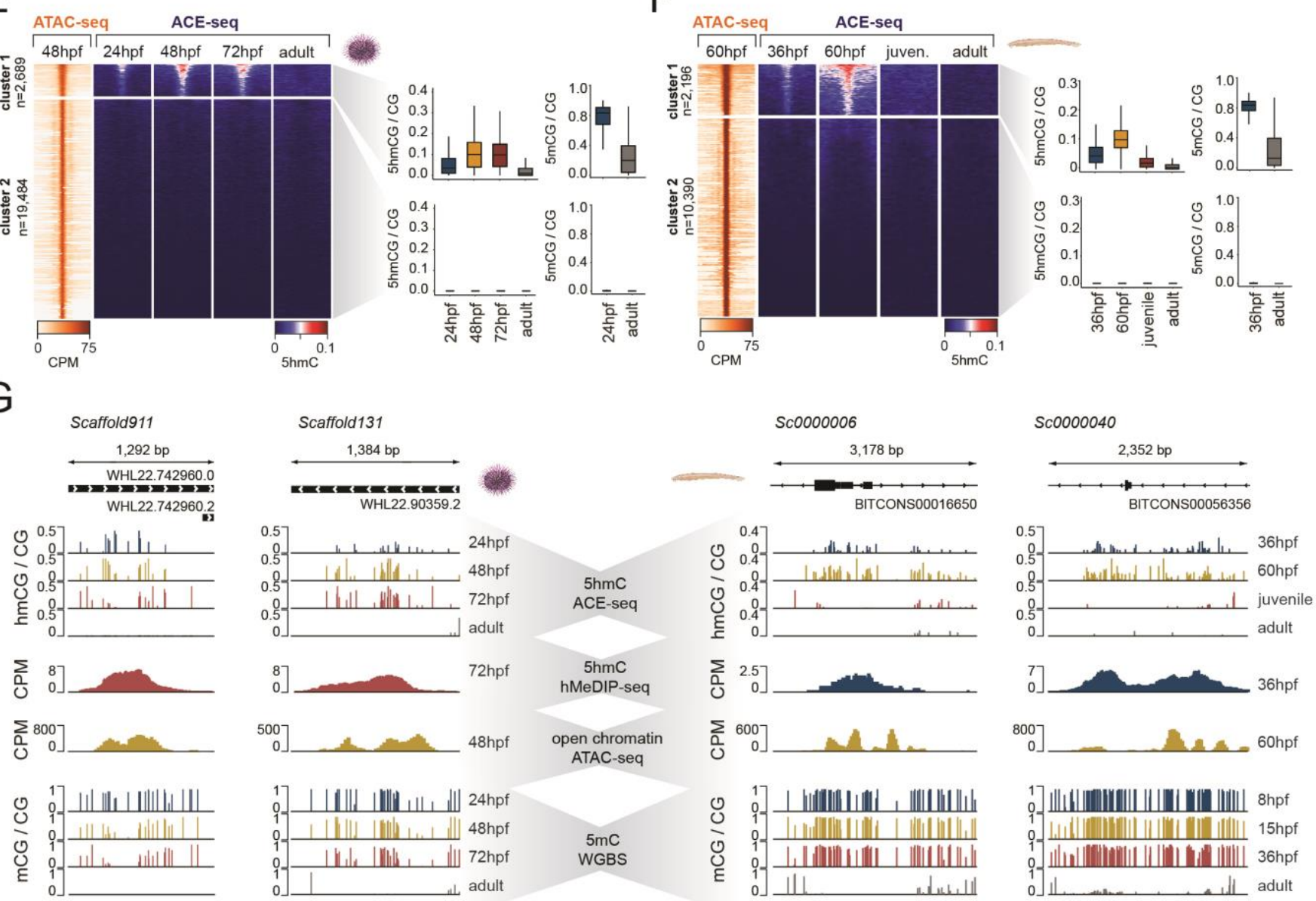

Figure 3. DNA hydroxymethylation landscape of sea urchin and lancelet development. (A) Percentage of methylated and hydroxymethylated CpGs in embryonic and adult tissues of sea urchin, lancelet, and zebrafish. CpG sites with at least $80 \%$ methylation and $\mathrm{CpG}$ sites with at least $10 \%$ hydroxymethylation $(P$ adj. $<0.05)$ were included in the analysis. Only $\mathrm{CpG}$ sites with a minimum of 10x coverage were considered. (B) Concordance of hydroxymethylation levels between biological replicates. Sea urchin $48 \mathrm{hpf}$ and lancelet 60hpf samples are shown. (C, D) Ternary plots depicting the relationship between methylated $(\mathrm{mC})$, 
hydroxymethylated (hmC) and unmethylated (C) $\mathrm{CpG}$ states in sea urchin (C) and lancelet (D) embryos and adult tissues. CpG sites hydroxymethylated at $48 \mathrm{hpf}$ in sea urchin $(\mathbf{C})$ and $60 \mathrm{hpf}$ in lancelet $(\mathbf{D})(P$ adj. $<0.05$, proportion test), are shown. (E, F) Heatmaps depicting DNA hydroxymethylation dynamics (ACE-seq) of open chromatin regions (ATAC-seq) in sea urchin (E) and lancelet (F). $\mathrm{K}$-means clustering $(\mathrm{k}=2)$ of ACE-seq and ATAC-seq signal over ATAC-seq peaks. Boxplots of DNA hydroxymethylation and DNA methylation dynamics during sea urchin (E) and lancelet (F) development. (G) IGV browser tracks depicting DNA hydroxymethylation (ACE-seq and hMeDIP-seq) enrichment at open chromatin regions (ATAC-seq) coinciding with DNA demethylation (MethylC-seq) in sea urchin and lancelet genomes.

methylated CpGs were in the $5 \mathrm{mC}$ state before the mid-developmental period. The same fraction also displayed a notable enrichment in unmethylated cytosines in adult tissues, in agreement with developmental $5 \mathrm{mC}$ removal. These data thus demonstrate that ACE-seq can accurately detect $5 \mathrm{hmC}$ at base resolution in sea urchin and lancelet genomes and that $5 \mathrm{hmC}$ displays comparable developmental dynamics across deuterostomes.

\section{5-hydroxymethylcytosine marks regulatory regions in deuterostome genomes}

Next, we wanted to assess to what extent does $5 \mathrm{hmC}$ correlate with sites of developmental hypomethylation and regulatory regions in general, identified through open chromatin profiling. We first plotted the $5 \mathrm{hmC}$ signal over previously identified adult hypomethylated DMRs (Fig. 2) and found a notable overlap between the two datasets (Fig. S3D). Out of 16,753 DMRs, 5,585 (33\%) formed an 5hmCenriched cluster in the sea urchin, whereas in the lancelet that overlap was $46 \%(2,072$ out of 4,507 DMRs). To obtain more comprehensive insight into the relationships between $5 \mathrm{hmC}$ and gene regulation, we generated open chromatin profiles (ATAC-seq), corresponding to $48 \mathrm{hpf}$ (sea urchin) and 60hpf (lancelet) ${ }^{38}$ stages (Fig. 3E, F), which are associated with peaks of TET activity (Fig. 1D). K-means clustering $(\mathrm{k}=2)$ of $5 \mathrm{hmC}$ signal over those regions revealed a subset (cluster 1), which was enriched for 5 hmC (Fig. 3E, F). This cluster was characterised by high $5 \mathrm{mC}$ levels in embryonic and low $5 \mathrm{mC}$ levels in adult tissues, in agreement with active demethylation occurring at these regions. $5 \mathrm{hmC}$ was highly enriched in $48 \mathrm{hpf}$ and 60hpf embryos in sea urchin and lancelet, respectively, and was almost undetectable in adult samples, as expected from the low $5 \mathrm{mC}$ signal in those tissues (Fig. 3E-G, Fig. S3E, F). Altogether, these results demonstrate strong $5 \mathrm{hmC}$ presence at invertebrate regulatory regions, which coincides with the active removal of $5 \mathrm{mC}$.

\section{5-hydroxymethylcytosine and developmental gene activation}

Having characterised developmental dynamics of 5hmC (Fig. 3), we next sought to understand which gene groups are potentially regulated by $5 \mathrm{hmC}$ and what impact might $5 \mathrm{hmC}$ have on developmental gene regulation. We first generated gene ontology (GO) profiles $^{59}$ of genes associated with $5 \mathrm{hmC}$ marked ATAC-seq peaks ${ }^{60}$ and found significant enrichment for developmental processes (Fig. 4A). Many of these processes such as "system development", "cell differentiation", "tissue development", "developmental process", and others, were identical to those associated with $5 \mathrm{hmC}$ regulated genes previously described in zebrafish, Xenopus, and mouse embryos ${ }^{19}$. We then assessed the genomic distribution of $5 \mathrm{hmC}$ enrichment and found that the majority of $5 \mathrm{hmC}$ peaks reside in gene bodies, even though a significant fraction of $5 \mathrm{hmC}$ was observed in promoters and intergenic regions (Fig. 4B). In line with their proposed developmental function, genes with both promoter and gene body $5 \mathrm{hmC}$ peaks were on average longer, suggestive of their increased regulatory potential (Fig. 4C, Fig. S4A, B $)^{61}$. Transcription factor binding motif analyses revealed significant enrichment for Sox and Fox binding sites, both of which have been previously shown to associate with TET proteins and DNA demethylation in vertebrates ${ }^{62,63}$ (Fig. 4D, Fig. S4C, D, Tables S8-13). In vertebrates, promoter $5 \mathrm{hmC}$ is linked to transcriptional activation ${ }^{64,65}$. To test whether a similar association could be reproduced in sea urchin and lancelet genomes, we analysed the transcriptional profiles of genes with a promoter $5 \mathrm{hmC}$ peak and found that promoter $5 \mathrm{hmC}$ correlates with activation of developmental genes in both examined organisms (Fig. 4E). A similar positive correlation between $5 \mathrm{hmC}$ and transcription was also observed at genes with gene body $5 \mathrm{hmC}$ peaks, however this effect was less pronounced, likely due to challenges in associating distal $5 \mathrm{hmC}$ peaks with their target genes (Fig. S4E). In sum, these results provide proof for the presence of $5 \mathrm{hmC}$ 
bioRxiv preprint doi: https://doi.org/10.1101/2021.11.07.467601; this version posted November 7, 2021. The copyright holder for this preprint (which was not certified by peer review) is the author/funder, who has granted bioRxiv a license to display the preprint in perpetuity. It is made available under aCC-BY 4.0 International license.

A
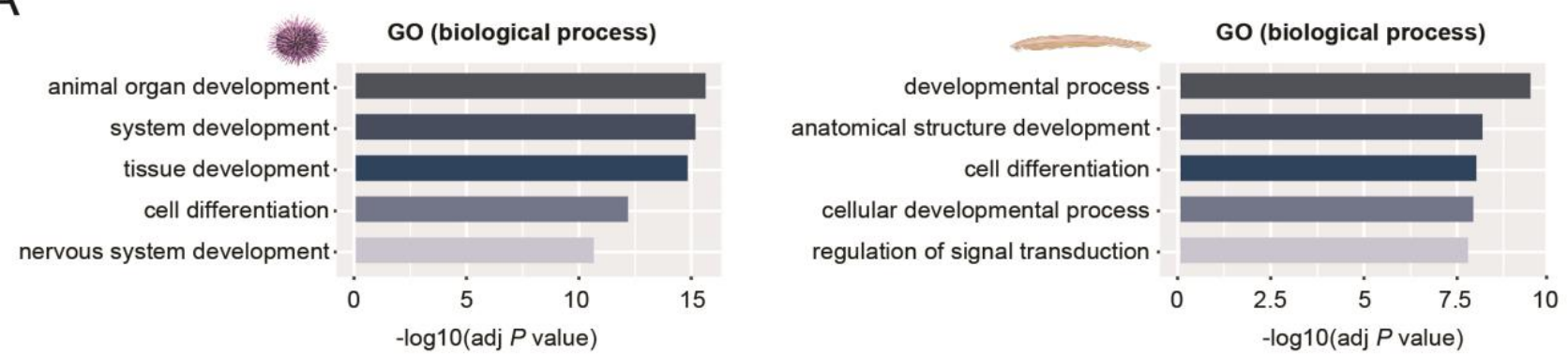

B

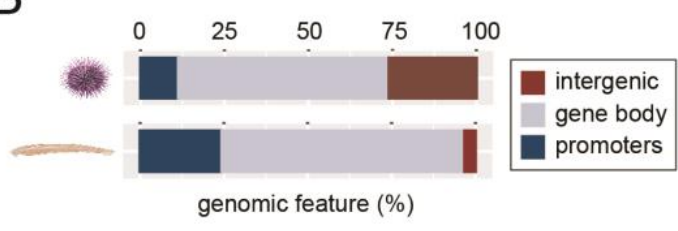

D

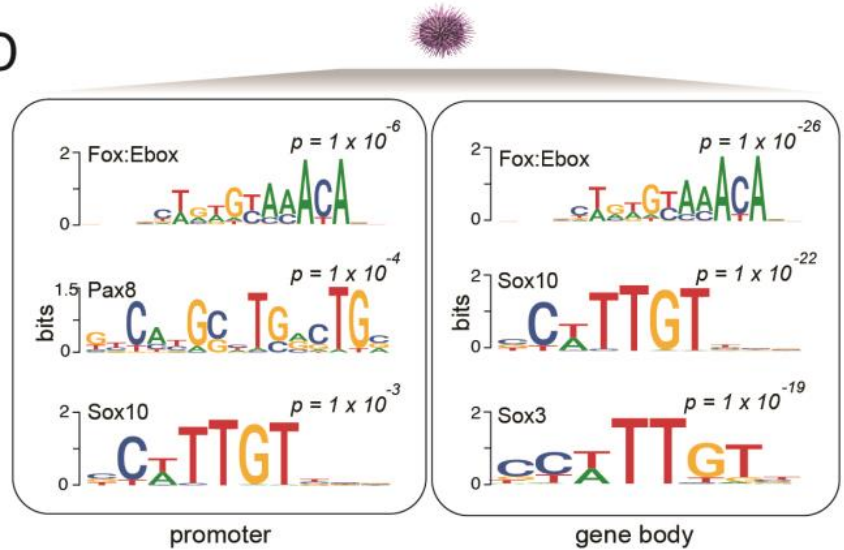

$\mathrm{E}$

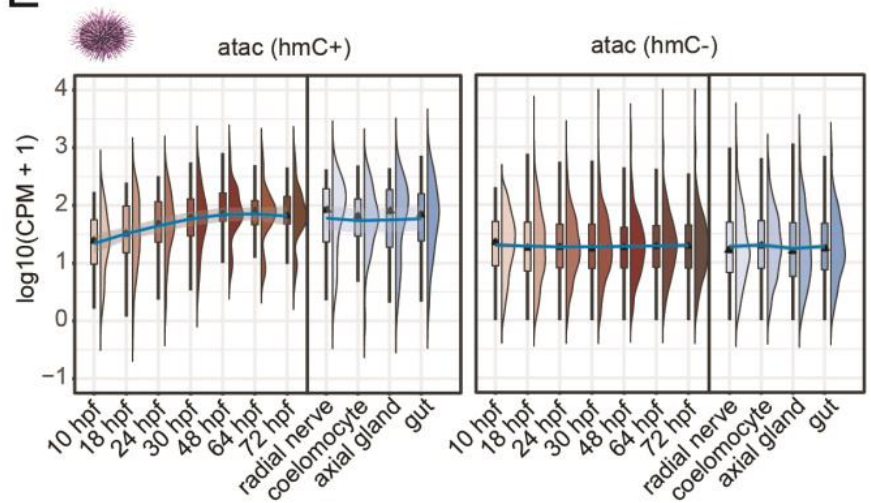

C

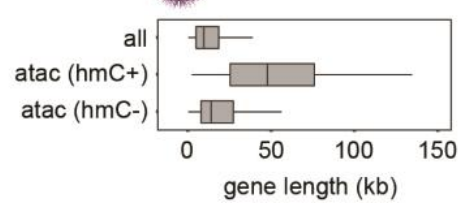

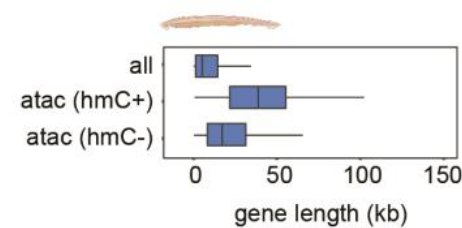

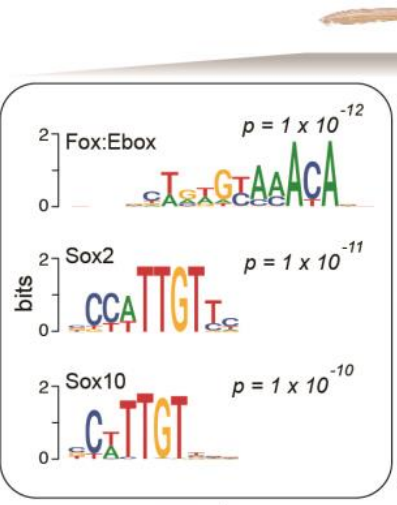

promoter

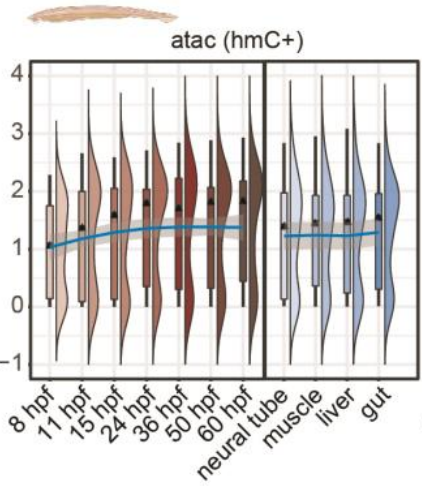

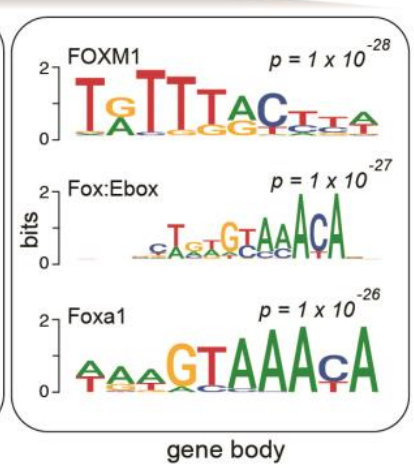

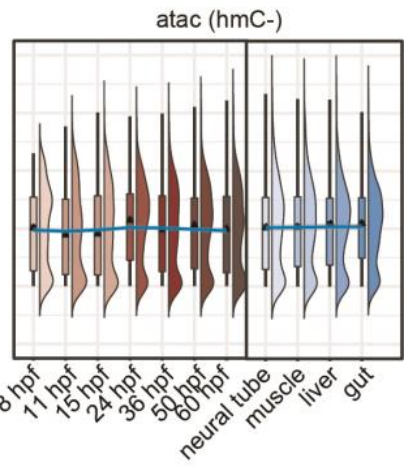

Figure 4. Developmental expression of $5 \mathrm{hmC}$-marked genes. (A) Gene ontology analysis of genes harbouring $5 \mathrm{hmC}$-marked ATAC-seq peaks at their promoters or gene bodies in sea urchin and lancelet. (B) Percentage of $5 \mathrm{hmC}$-marked ATAC-seq peaks at different genomic regions. (C) Length of the genes harbouring promoter-associated $5 \mathrm{hmC}$-marked ATAC-seq peaks (promoter atac $\mathrm{hmC}+$ ) and non-5hmC ATAC-seq peaks (promoter atac hmC-). (D) HOMER motif enrichment analysis of atac hmC+ regions associated with either gene promoters or gene bodies. (E) Expression dynamics of promoter atac hmC+ genes as compared to promoter atac hmC- genes in sea urchin and lancelet embryos and adult tissues.

at regulatory regions of developmental genes in invertebrate deuterostome genomes and suggest that active removal of $5 \mathrm{mC}$ through the $5 \mathrm{hmC}$ intermediate might be crucial for their activation.

\section{Conserved regulatory logic of 5-hydroxymethyl- cytosine-marked genes}

Previously, we have described widespread TETmediated demethylation of regulatory regions during the vertebrate phylotypic period, which can be 
defined as the most conserved stage of vertebrate embryogenesis ${ }^{19}$. These phylotypic DMRs (phyloDMRs), coincided with developmental DNA demethylation and were marked by $5 \mathrm{hmC}$ in zebrafish, Xenopus and mouse embryos. To explore the extent to which genes regulated by $5 \mathrm{hmC}$ in invertebrate deuterostomes coincide with genes linked to vertebrate $5 \mathrm{hmC}$, we first identified zebrafish orthologues of $5 \mathrm{hmC}$-marked genes in sea urchin and lancelet, defined their regulatory landscapes ${ }^{60}$, selected the zebrafish orthologs enriched in 5hmC, and assessed their overlap (Fig. S5A). This analysis resulted in 148, and 343 zebrafish $5 \mathrm{hmC}$-marked genes, which overlapped lancelet and sea urchin 5hmC-marked genes, respectively. Having identified this notable agreement in $5 \mathrm{hmC}$ regulation, we next wanted to explore in more detail the regulatory logic behind $5 \mathrm{hmC}$ gene marking. To that end we selected zebrafish orthologues of sea urchin 5hmC-marked genes and stratified ATAC-seq-associated regulatory elements in the zebrafish genome within each gene's landscape $^{60}$ into three categories depending on phylo-DMR presence and 5hmC enrichment (Fig. S5B): group 1 (ATAC-seq peaks overlapping a phylo-DMR and displaying 5hmC enrichment), group 2 (ATAC-seq peaks displaying $5 \mathrm{hmC}$ enrichment without phylo-DMR overlap), and group 3 (ATAC-seq peaks without $5 \mathrm{hmC}$ enrichment or phylo-DMR overlap). We next explored the transcriptional profiles of zebrafish orthologs linked to these three groups and found that phylo-DMRlinked genes (group 1) were characterised by peaks of expression surrounding the phylotypic period (24hpf), whereas 5hmC-marked genes without phylo-DMR association (group 2) were developmentally upregulated at later embryonic stages (4872hpf) and exhibited strongest expression in the adult brain (Fig. 5A). Genes that were not enriched in $5 \mathrm{hmC}$ or phylo-DMRs (group 3) did not display such notable developmental dynamics (Fig. 5A). To obtain further insight into the spatio-temporal regulation of these orthologs, we analysed single cell resolution transcriptomes of the zebrafish phylotypic stage embryo (24hpf) (Fig. 5B $)^{66}$. We found that group 1 genes were expressed broadly in cell-types originating from diverse embryonic layers, whereas group 2 genes were expressed predominantly in neurons, in line with their bulk RNA-seq expression profile (Fig. 5A-C, Fig. S5C). Group 3 orthologues were characterised by a minor enrichment in the erythroid lineage. Given the strong link between $5 \mathrm{hmC}$ and loss of $5 \mathrm{mC}$ in non-vertebrate (Fig. 2) and vertebrate $^{19}$ adult tissues, we next sought to interrogate the $5 \mathrm{mC}$ state of these three groups of regulatory elements in adult zebrafish ${ }^{67}$ (Fig. 5D). We found that both $5 \mathrm{hmC}$-enriched ATAC peaks with and without phylo-DMR association are predominantly hypomethylated in most adult tissues (Fig. 5D). Unlike group 2 elements, which are exclusively hypomethylated in adults, group 1 elements were already hypomethylated during the phylotypic period. Finally, group 3 elements that displayed no $5 \mathrm{hmC}$ enrichment coincided with peaks of broad and pervasive hypomethylation characteristic of $\mathrm{CpG}$ islands ${ }^{68}$. These data argue that most invertebrate deuterostome $5 \mathrm{hmC}$-marked genes also display an association with this regulatory mark in vertebrates, which utilise $5 \mathrm{hmC}$ during organogenesis (group 1 elements) as well as during later stages of tissue differentiation (group 2 elements).

\section{Discussion}

The goal of the current study was to explore the evolutionary conservation of TET-mediated DNA demethylation in non-vertebrate lineages by generating base-resolution profiles of developmental 5-hydroxymethylcytosine landscape dynamics. Firstly, we have shown that the expression of sea urchin and lancelet TET orthologues by and large resembles anamniote TET developmental expression profiles, which are characterised by peaks of TET activity during mid-development (phylotypic period $)^{19}$. While TET proteins play important roles in mammalian pluripotency and reprogramming in vitro $^{21,64,65,69}$, mouse triple TET knockouts developed normally until gastrulation ${ }^{18}$ indicative of reduced requirements for TET activity during in vivo pluripotency. Similarly, zebrafish homozygous TET knockouts generated by TALE technology ${ }^{22}$, or CRISPR/Cas9 F0 mutants ${ }^{20}$ and morphants ${ }^{19}$, all displayed developmental defects only during later embryonic stages. Our results thus suggest that active DNA demethylation removal and likely $5 \mathrm{mC}$ in general, are not required for the establishment of deuterostome pluripotency. 
bioRxiv preprint doi: https://doi.org/10.1101/2021.11.07.467601; this version posted November 7, 2021. The copyright holder for this preprint (which was not certified by peer review) is the author/funder, who has granted bioRxiv a license to display the preprint in perpetuity. It is made available under aCC-BY 4.0 International license.

A

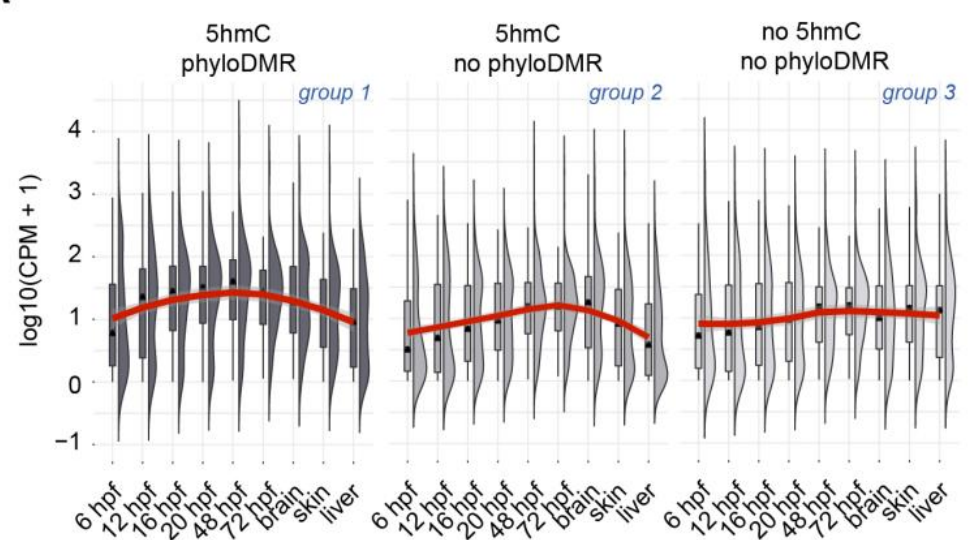

$\mathrm{B}$

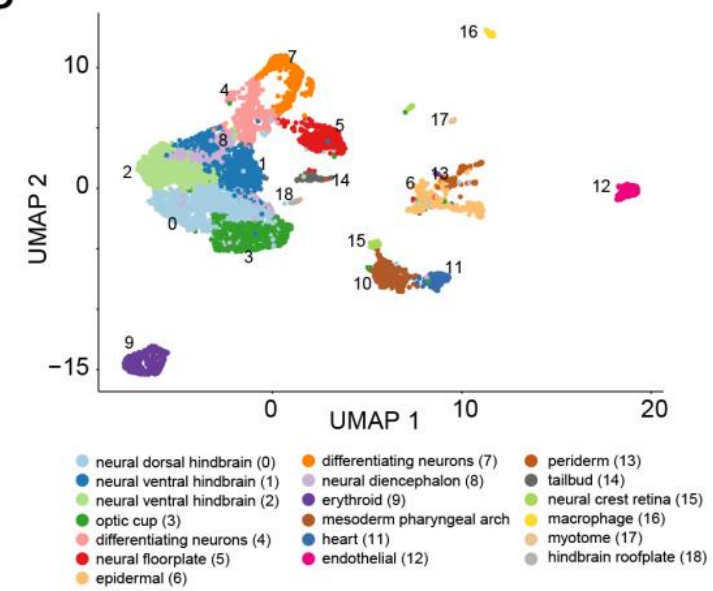

C

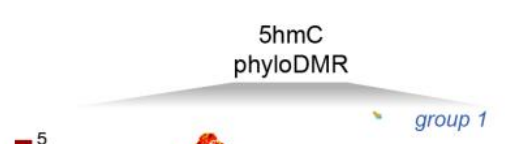

I

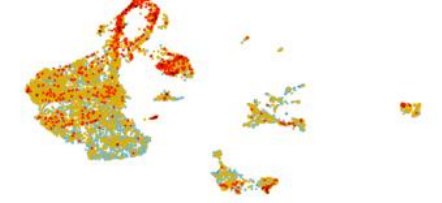

$5 \mathrm{hmC}$ no phyloDMR

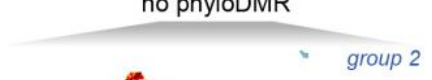

$5 \mathrm{hmC}$

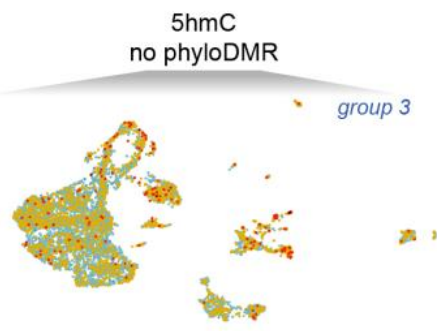

(4)

$\mathrm{D}$

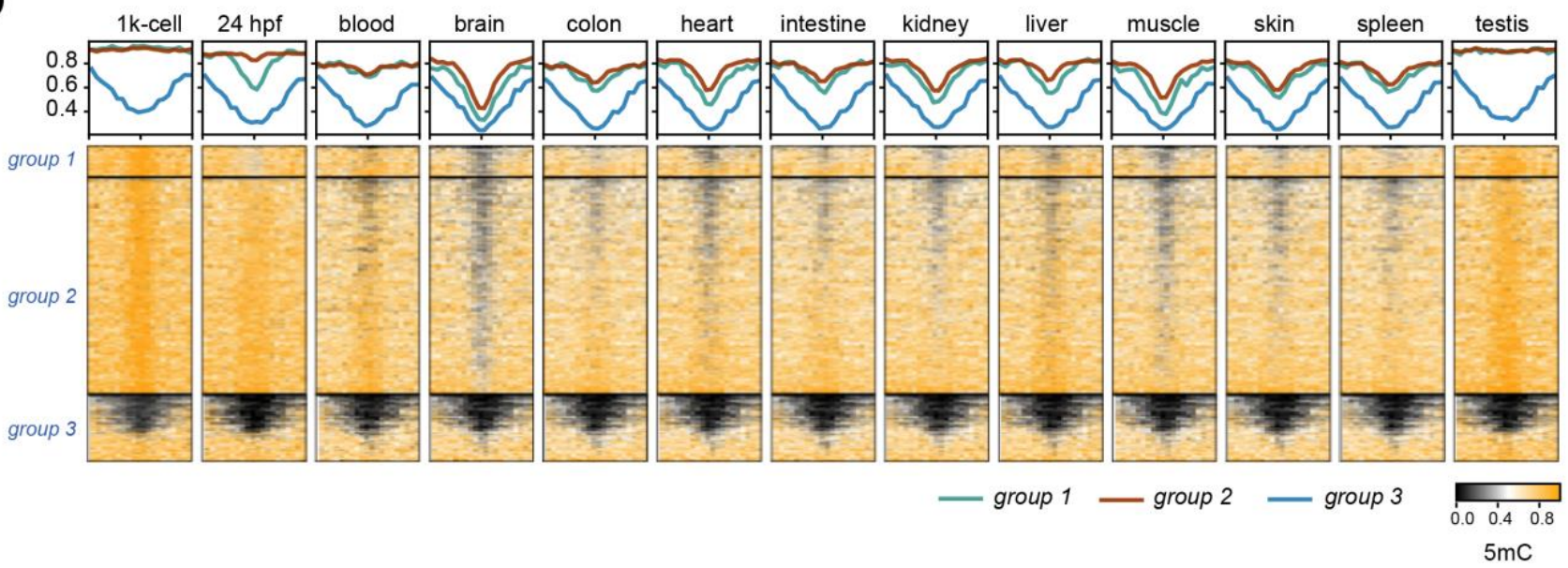

Figure 5. Conservation of developmental regulatory logic of 5 hmC-marked genes. (A) Expression of 2R/3R-ohnologue zebrafish genes either associated with 5hmC-marked ATAC-seq peaks overlapping phylo-DMRs (5hmC/phylo-DMR), 5hmCmarked ATAC-seq peaks not overlapping phylo-DMRs (5hmC/no phylo-DMR) or non-5hmC ATAC-seq peaks not overlapping phylo-DMRs (no 5hmC/no phylo-DMR) in embryos and adult tissues. (B) Uniform Manifold Approximation and Projection (UMAP) projection of 7,738 individual cells from the 24hpf zebrafish embryo. Cells are colour-coded by tissue types. (C) ztransformed module scores of three gene sets: $5 \mathrm{hmC} / \mathrm{phylo}-\mathrm{DMR}, 5 \mathrm{hmC} /$ no phylo-DMR and no $5 \mathrm{hmC} / \mathrm{no} \mathrm{phylo}-\mathrm{DMR}$ in $24 \mathrm{hpf}$ zebrafish embryo. Module scores depict the difference between the average gene expression of each gene set and random control genes. (D) Heatmaps showing DNA methylation profiles of $5 \mathrm{hmC} / \mathrm{phyloDMR}, 5 \mathrm{hmC} / \mathrm{no}$ phyloDMR and no $5 \mathrm{hmC} / \mathrm{no}$ phyloDMR ATAC-seq peaks in $1 \mathrm{~K}$-cell and $24 \mathrm{hpf}$ zebrafish embryos as well as multiple adult tissues.

While the members of TET/JBP (J-protein binding) family have been identified across all domains of life, ranging from viruses to humans ${ }^{33}$, fairly little is known regarding the functions of these proteins in DNA demethylation beyond vertebrates $28,35,37$. For example, in Drosophila, the single identified TET orthologue has only been associated with RNA hydroxymethylation ${ }^{34}$ whereas detailed, baseresolution invertebrate 5-hydroxymethylcytosine maps have thus far only been generated for the demosponge A. queenslandica and the sea anemone Nematostella vectensis ${ }^{31}$. Interestingly however, in A. queenslandica, $5 \mathrm{hmC}$ was detectable at identical positions during embryonic and larval stages without 
displaying any signs of developmental loss ${ }^{28}$. This suggests that $A$. queenslandica either uses $5 \mathrm{hmC}$ as a stable gene-regulatory mark or that TET-mediated DNA demethylation occurs during later developmental transitions. In this work we conclusively demonstrate that genomic sites of $5 \mathrm{hmC}$ enrichment coincide with developmental $5 \mathrm{mC}$ loss and the presence of accessible chromatin, in line with the proposed roles of TET proteins and $5 \mathrm{hmC}$ in gene regulation ${ }^{70}$. Specifically, we detect $5 \mathrm{hmC}$ at both proximal and distal regulatory regions and show that $5 \mathrm{hmC}$ presence and developmental loss of $5 \mathrm{mC}$ coincide with activation of developmental genes. We have previously demonstrated that active DNA demethylation of enhancers associated with key developmental pathways is a hallmark of vertebrate development ${ }^{19}$. Here we further expand on these observations to conclude that TET activity and active DNA demethylation of regulatory regions during mid-developmental stages could form a conserved developmental signature of diverse animal phyla. It is worth noting however, that the current study is limited to invertebrate deuterostomes from the phylum Echinodermata (S. purpuratus) and Chordata (B. lanceolatum), making it thus challenging to extrapolate our findings beyond these two lineages. Deeply conserved DNA demethylation toolkits consisting of TET proteins and DNA repair machinery as well as canonical gene body $5 \mathrm{mC}$ patterns observed in most animals ${ }^{28,33}$ would support widespread usage of TETs and $5 \mathrm{hmC}$ for gene regulation. Nevertheless, transcriptome profiling of multiple developmental stages across ten different phyla demonstrated divergence in the usage of signalling pathways and transcription factors during mid-development ${ }^{71}$. It remains to be seen how prevalent TET-mediated regulation of developmental genes is in other animal lineages, and if $5 \mathrm{mC} / 5 \mathrm{hmC}$ regulatory logic could be ancestral to bilaterian animals. Finally, in the current study we have shown that $5 \mathrm{hmC}$-regulated genes identified in invertebrate deuterosteomes also display $5 \mathrm{mC}$ and $5 \mathrm{hmC}$-mediated regulation in the zebrafish genome and that most of such $5 \mathrm{hmC}$-enriched genes are either linked to actively demethylated phylotypic enhancers or regulatory regions that become demethylated in diverse adult tissues, and in particular adult brain. Overall, our study provides novel insight into the $5 \mathrm{mC} / 5 \mathrm{hmC}$ regulatory logic beyond the invertebrate-vertebrate boundary and demonstrates that active $5 \mathrm{mC}$ removal from regulatory regions during animal development predates vertebrate origins.

\section{Methods \\ Genomic DNA extraction from sea urchin embryos and adult tissues}

Strongylocentrotus purpuratus adult animals were collected in the San Diego Bay area, San Diego County, California (USA) and kept in a closed tank system with circulating diluted Mediterranean Sea water at $36 \mathrm{ppt}$ and $14^{\circ} \mathrm{C}$ at Stazione Zoologica Anton Dohrn. Adult tube feet were collected by surgically removing them from the adult sea urchin attached to a glass beaker with sea water. To obtain embryos and larvae, sea urchin gametes were collected by vigorously shaking the adults. Eggs were collected by placing the sea urchin aboral side up onto a beaker filled with seawater. Sperm was collected with a p200 micropipette into a $1.5 \mathrm{~mL}$ tube, which was placed on ice. The eggs were filtered once through a $100 \mu \mathrm{m}$ mesh filter to remove debris. $5 \mu \mathrm{L}$ of sperm was diluted in $13 \mathrm{~mL}$ of seawater, then 20 drops of this dilution were added to the eggs to fertilize them. Fertilized eggs were incubated in seawater at $15^{\circ} \mathrm{C}$ until the desired stage. $25-50 \mu \mathrm{L}$ of sea urchin larvae or embryos were collected for DNA extraction by using a $40 \mu \mathrm{m}$ mesh filter and placed into $1.5 \mathrm{~mL}$ tubes. Tubes with adult tube feet, embryos and larvae were centrifuged at $500 \mathrm{~g}$ and the supernatant was discarded. The resulting pellets were frozen by placing the tubes in liquid nitrogen and then stored at $-80^{\circ} \mathrm{C}$ until further processing. The DNA extraction procedure was identical for sea urchin adult tube feet, embryos, and larvae. The frozen pellet was washed 3 times with $1 \mathrm{X} \mathrm{SSC}$ in milliQ $\mathrm{H} 2 \mathrm{O}$ by centrifuging at $500 \mathrm{x}$ g for 5 minutes and removing the supernatant. The washed pellet was resuspended in $750 \mathrm{~mL}$ of lysis buffer $(10 \mathrm{mM}$ Tris $\mathrm{pH} 7.5,1 \mathrm{mM}$ EDTA, $1 \%$ SDS, $3.4 \% \beta-$ mercaptoethanol, $660 \mu \mathrm{g} / \mathrm{mL}$ Proteinase $\mathrm{K}$ ) and incubated at $56^{\circ} \mathrm{C}$ for 2 hours at $300 \mathrm{rpm}$ in a heat block. After cooling down, an equal amount of PCI (Phenol/Chlorophorm/Isoamylalcohol mixture in 25:24:1 proportion at neutral $\mathrm{pH}$ ) was added and mixed by inverting the tube several times, followed by centrifugation at $16,000 \mathrm{x}$ for 10 minutes at $4{ }^{\circ} \mathrm{C}$ to separate the phases. The top phase was collected and mixed with an equal volume of CIA (Chloroform/Isoamyl Alcohol mixture in 24:1 
proportion). The solution was mixed by inversion and centrifuged at $16,000 \mathrm{xg}$ for 10 minutes at $4^{\circ} \mathrm{C}$. The top phase was collected and combined with 0.1 volume of $3 \mathrm{M} \mathrm{NaAc}(\mathrm{pH} \mathrm{5.0)}$ ) and 2 volumes of icecold $100 \%$ EtOH. The solution was mixed by inverting the tube several times. Precipitated DNA was collected by a p20 micropipette and placed in a $1.5 \mathrm{~mL}$ tube with $200 \mu \mathrm{L}$ of $70 \% \mathrm{EtOH}$. This procedure was repeated twice to wash the DNA. The precipitated DNA pellet was then placed into an empty clean $1.5 \mathrm{~mL}$ tube and air-dried to remove EtOH. Dried DNA was resuspended in 200 to 500 $\mu \mathrm{L}$ of $1 \mathrm{x}$ TE buffer ( $\mathrm{pH}$ 8.0) depending on the amount of DNA.

\section{Genomic DNA extraction from lancelet embryos and adult tissues}

Adults of Branchiostoma lanceolatum were collected at the Racou beach near Argelès-sur-Mer, France (latitude $42^{\circ} 32^{\prime} 53^{\prime \prime} \mathrm{N}$, longitude $3^{\circ} 03^{\prime} 27^{\prime \prime}$ E) with a specific permission delivered by the Prefect of Region Provence Alpes Côte d'Azur. Branchiostoma lanceolatum is not a protected species. Gametes were obtained after heat stimulation as previously described ${ }^{72}$ and were used for in vitro fertilization in Petri dishes filled with filtered seawater. Embryos were collected at the desired stages and placed in $1.5 \mathrm{~mL}$ tubes (at least 500 embryos per tube). The seawater was discarded after 1 minute of centrifugation at $13,000 \mathrm{rpm}$. Adults were immobilized in $\mathrm{MgCl}_{2} 7 \%$ for 5 minutes, placed in seawater and sacrificed by decapitation. The hepatic caecum was dissected and placed in $1.5 \mathrm{~mL}$ tubes. The embryos and adult tissues were processed using the DNeasy Blood and Tissue kit (QIAGEN) following the instructions provided by the manufacturer, with an incubation in lysis buffer at $56^{\circ} \mathrm{C}$ for 5 hours and using $200 \mu \mathrm{L}$ of elution buffer.

\section{MethylC-seq library preparation}

MethylC-seq library preparation was performed as described previously ${ }^{40}$. Briefly, $1000 \mathrm{ng}$ of genomic DNA extracted from sea urchin and lancelet embryos and adult tissues was spiked with unmethylated $\lambda$ phage DNA (Promega) and sonicated to $\sim 300 \mathrm{bp}$ fragments using a M220 focused ultrasonicator (Covaris) with the following parameters: peak incident power, $50 \mathrm{~W}$; duty factor, $20 \%$; cycles per burst, 200; treatment time, $75 \mathrm{sec}$. Sonicated DNA was then purified, end-repaired using End-It ${ }^{\mathrm{TM}} \mathrm{DNA}$ End-Repair Kit (Lucigen) and A-tailed using Klenow Fragment $\left(3^{\prime} \rightarrow 5^{\prime}\right.$ exo-) (New England Biolabs) followed by the ligation of NEXTFLEX ${ }^{\circledR}$ Bisulfite-Seq adapters. Bisulfite conversion of adaptor-ligated DNA was performed using EZ DNA Methylation-Gold Kit (Zymo Research). Library amplification (13 PCR cycles) was performed with KAPA HiFi HotStart Uracil+ DNA polymerase (Kapa Biosystems). Library size was determined by the Agilent 4200 Tapestation system. The libraries were quantified using the KAPA library quantification kit (Roche), yielding $\sim 10-20 \mathrm{nM}$.

\section{ACE-seq library preparation}

ACE-seq library preparation for base resolution 5hydroxymethylcytosine profiling was performed as previously described ${ }^{41,73}$. Briefly, 100 ng of genomic DNA extracted from sea urchin, lancelet, and zebrafish embryos and adult tissues was spiked with $1 \mathrm{ng}$ of CpG-methylated $\lambda$ phage DNA (Wisegene) and $0.5 \mathrm{ng}$ of all-C hydroxymethylated pUC19 plasmid DNA (Wisegene) and sonicated to $\sim 300 \mathrm{bp}$ fragments using a M220 focused ultrasonicator (Covaris), with the following parameters: peak incident power, 50W; duty factor, $20 \%$; cycles per burst, 200; treatment time, $75 \mathrm{sec}$. Sonicated DNA (125 $\mu \mathrm{L}$ total volume) was concentrated to $16.6 \mu \mathrm{L}$ using AMPure beads (1 volume DNA : 2 volumes AMPure beads). $5 \mathrm{hmC}$ protection reaction was performed using $\beta$-glucosyltransferase $(10 \mathrm{U} / \mu \mathrm{L}$, New England Biolabs) in Cutsmart Buffer according to manufacturer's instructions at $37^{\circ} \mathrm{C}$ for $1 \mathrm{hr}$, followed by a $10 \mathrm{~min}$ incubation at $65^{\circ} \mathrm{C}$. The DNA was denatured in DMSO at $95^{\circ} \mathrm{C}$ for $10 \mathrm{~min}$ and immediately placed on dry ice for 2 mins allowing the samples to freeze. $\mathrm{C}$ and $5 \mathrm{mC}$ deamination reaction was performed using the APOBEC3A enzyme (NEBNext ${ }^{\circledR}$ Enzymatic Methyl-seq Kit, New England Biolabs) with the following ramping conditions: $4^{\circ} \mathrm{C}$ for $10 \mathrm{~min}, 4^{\circ} \mathrm{C}-50^{\circ} \mathrm{C} 2: 15 \mathrm{~min}$ per degree of the ramp, $50^{\circ} \mathrm{C}$ for $10 \mathrm{~min}$. Deaminated DNA was then purified using AMPure beads (1 volume DNA: 1 volume AMPure beads) and subjected to low input library preparation using Accel-NGS Methyl-Seq DNA kit (Swift Biosciences). Briefly, DNA was denatured and subjected to an adaptase reaction, followed by primer extension, adapter ligation, and 15 cycles of indexing PCR. Library size and consistency was determined 
by the Agilent 4200 Tapestation system. The libraries were quantified using the KAPA library quantification kit (Roche).

\section{hMeDIP-seq library preparation}

The hMeDIP assay was performed according to the manufacturer's instructions (Active Motif, hMeDIP, Cat No 55010) on sea urchin 72hpf, lancelet 36hpf, and zebrafish $24 \mathrm{hpf}$ embryos. DNA was sonicated with the Covaris sonicator to generate $\sim 300 \mathrm{bp}$ fragments. Briefly, 200-400 ng of fragmented DNA was spiked with either unmethylated, $5 \mathrm{mC}$ methylated or 5hmC hydroxymethylated 338-bp PCR product of APC genomic locus. The DNA was denatured for $10 \mathrm{~min}$ at $95^{\circ} \mathrm{C}$ and immunoprecipitated overnight at $4^{\circ} \mathrm{C}$ with $4 \mu \mathrm{L}$ of $5 \mathrm{hmC}$ monoclonal antibody (Active Motif Cat No 55010). To allow selective enrichment of immunecaptured DNA fragments, the mixture was incubated with $25 \mu \mathrm{L}$ of Protein $\mathrm{G}$ magnetic beads for $2 \mathrm{~h}$ at $4^{\circ} \mathrm{C}$ prior to washing of unbound DNA fragments. Bound hydroxymethylated DNA was eluted, treated with proteinase $\mathrm{K}$ and purified by Phenolchloroform/isoamyl alcohol extraction and ethanol precipitation. hmC-containing DNA was subjected to library preparation using the TruSeq ChIP Sample Preparation kit, (Illumina). The specificity of the hMeDIP assay was validated by qPCR of the unmethylated, methylated and hydroxymethylated spike-in APC controls.

\section{ATAC-seq library preparation}

ATAC-seq assays were performed on sea urchin embryos as previously described ${ }^{74,75}$. For each of the two ATAC-seq replicates, approximately 45,000 cells (corresponding to $\sim 135$ embryos at $48 \mathrm{hpf}$ ) were lysed in cold lysis buffer (10 mM Tris $\mathrm{pH} 7.4$, $10 \mathrm{mM} \mathrm{NaCl}, 3 \mathrm{mM} \mathrm{MgCl} 2$ and $0.2 \%$ NP40/Igepal) after removing the seawater and washing the embryos with filtered ice-cold artificial seawater three times by centrifuging at $500 \mathrm{x}$. The resultant nuclei were then incubated for $30 \mathrm{~min}$ at $37^{\circ} \mathrm{C}$ with an in-house made Tn5 enzyme and purified with the Qiagen MinElute kit (Qiagen, 28004). PCR reactions for each replicate were performed with 15 cycles using Ad1F and Ad2.2R primers and NEBNext High-Fidelity 2X PCR Master Mix (New England Labs Cat \#M0541). The resulting libraries were multiplexed and sequenced on the HiSeq 4000 sequencer.

\section{MethylC-seq data analysis}

Sea urchin and lancelet MethylC-seq libraries were sequenced on the Illumina HiSeqX platform (150 bp, PE). Sequenced reads in FASTQ format were trimmed using Trimmomatic (ILLUMINACLIP:adapter.fa:2:30:10 SLIDINGWINDOW:5:20 LEADING:3 TRAILING:3 MINLEN:50). Trimmed reads were mapped to Spur_v3.1, B171nemr and danRer10 genome references for sea urchin, lancelet, and zebrafish, respectively, (all containing the $\lambda$ phage genome as chrLambda) using WALT $^{76}$ with the following settings: -m 10 -t $24-\mathrm{N} 10000000$-L 2000. Mapped reads in SAM format were converted to BAM and indexed using SAMtools ${ }^{77}$. Optical and PCR duplicates were removed using Picard Tools function MarkDuplicates REMOVE_DUPLICATES=true. Methylated and unmethylated cytosines at each genomic $\mathrm{CpG}$ position were called using MethylDackel v0.3.0. Additional MethylDackel extract parameters -minOppositeDepth 5 --maxVariantFrac 0.5 were used for the genotype correction (in order to exclude C-to-T nucleotide transitions). Additionally, sequencing read boundaries obtained from MethylDackel mbias were reviewed and fed into MethylDackel extract --OT a,b,c,d, --OB a,b,c,d parameters. For MethylC-seq, bisulfite conversion efficiency was estimated from the $\lambda$ phage spike-in control.

\section{ACE-seq data analysis}

Sea urchin, lancelet, zebrafish and mouse ACE-seq libraries were sequenced on Illumina HiSeqX and NovaSeq 6000 platforms (150 bp, PE). Adapter clipping and read quality trimming was performed using Trimmomatic (ILLUMINACLIP:NEXTflex.fa:2:30:10 SLIDINGWINDOW:5:20 LEADING:5 TRAILING:5 MINLEN:50 CROP:140 HEADCROP:10 $)^{78}$. These settings allow for the removal of low complexity Adaptase tails. Trimmed reads were mapped to Spur_v3.1, B171nemr, danRer10 and mm10 genome references for sea urchin, lancelet, zebrafish and mouse ${ }^{41}$, respectively, (all containing the $\lambda$ phage genome as chrLambda and pUC19 plasmid genome as chrPUC) using WALT $^{76}$ with the following settings: -m 10 -t 24 -N 10000000 -L 2000. Mapped reads in SAM format were converted to BAM and indexed using SAMtools ${ }^{77}$. Optical and PCR duplicates were 
removed using Picard Tools's function MarkDuplicates REMOVE_DUPLICATES $=$ true . Reads with more than three consecutive nonconverted cytosines in the CC, CA and CT context were removed using Picard Tools' function FilterSamReads. In order to determine sequencing read boundaries displaying uniform hydroxymethylation rate and to increase the accuracy of $\mathrm{CpG}$ hydroxymethylation calling, we ran the methylation bias command: MethylDackel mbias. The numbers of hydroxymethylated and unmethylated cytosines at each genomic $\mathrm{CpG}$ position were called using the MethylDackel extract genome.fasta input.bam -o output --mergeContext. Additional MethylDackel extract parameters -minOppositeDepth 5 --maxVariantFrac 0.5 were used for genotype correction. Additionally, sequencing read boundaries obtained from MethylDackel mbias were reviewed and fed into MethylDackel extract --OT a,b,c,d, --OB a,b,c,d parameters.

\section{Statistical analysis of hydroxymethylation calling}

Statistical analysis of the accuracy of ACE-seq $5 \mathrm{hmC}$ calling at a given $\mathrm{CpG}$ site was performed following the assumption that the number of " $\mathrm{C}$ " reads $\left(\mathrm{N}_{\mathrm{c}}\right)$ for each $\mathrm{CpG}$ site follows a binomial distribution $N_{c} \sim \operatorname{binomial}(p, n)$ where $p$ (probability distribution) is the $5 \mathrm{mC}$-to- $\mathrm{T}$ non-conversion rate and $n$ is sequencing depth (number of " $\mathrm{C} "+$ " $\mathrm{T}$ " reads per each $\mathrm{CpG}$ site). Proportion test ( $\mathrm{R}$ prop.test) was utilized to test the null hypothesis $\mathrm{H} 0$ that the observed proportion (the number of "C" reads at a given $\mathrm{CpG}$ site) equals the expected proportion (p) and the alternative hypothesis $\mathrm{H} 1$ that that the observed proportion is greater than the expected proportion. The following parameters were used: $\operatorname{prop}$.test $(x, n, p=$ nonconv_rate, alternative $=$ "greater"), where $x$ is a vector of counts of successes (number of "C" reads for each $\mathrm{CpG}$ site), $n$ - a vector of counts of trials (number of "C" + "T" reads per each $\mathrm{CpG}$ site, i.e coverage), $p$ - a vector of probabilities of success (5mC-to- $\mathrm{T}$ non-conversion rate). Non-conversion rate of each ACE-seq library was estimated using the M.SssI CpG methyltransferase $5 \mathrm{mCG}$-methylated $\lambda$ phage spikein controls. The non-conversion rate is the proportion of "C" reads at methylated $\mathrm{CpG}$ sites of the $5 \mathrm{mCG} \lambda$ phage spike-in control being incorrectly retained as cytosines due to inefficient APOBEC3A-mediated deamination of $5 \mathrm{mC}$. The statistical analysis was restricted to $\mathrm{CpG}$ sites covered by at least 10 reads.

\section{Identification of differentially methylated regions (DMRs)}

Differentially methylated $\mathrm{CpG}$ sites between consecutive embryonic and adult stages were identified using the DMLtest function of the DSS package $^{79}$. Differentially methylated $\mathrm{CpG}$ sites located within $50 \mathrm{bp}$ from each other were joined into regions (DMRs), which were filtered to harbour at least 5 differentially methylated $\mathrm{CpGs}$ that span at least $50 \mathrm{bp}$. DMRs containing more than $25 \%$ of CpGs within them with sequencing coverage less than $5 \mathrm{x}$ were excluded from the analysis. The remaining DMRs were defined as hypomethylated or hypermethylated when both replicates displayed at least $20 \%$ average methylation difference between consecutive developmental stages.

\section{hMeDIP-seq data analysis}

hMeDIP-seq libraries from sea urchin $72 \mathrm{hpf}$, lancelet 36hpf, and zebrafish $24 \mathrm{hpf}$ embryos were sequenced on Illumina HiSeq X platform. hMeDIPseq raw data was processed using the NGSANE framework v0.5.2. $0^{80}$. Briefly, sequenced hMeDIPseq reads in FASTQ format were trimmed using Trimmomatic (ILLUMINACLIP:TruSeq3PE.fa:2:30:10 SLIDINGWINDOW:5:20). Trimmed reads were mapped to Spur_v3.1, B171nemr and danRer10 reference genomes for sea urchin, lancelet and zebrafish, respectively, using bowtie 2 v2.1.0 with the following parameters: bowtie 2 --end-to-end $-R 2$-p 10 -N 1 --very-sensitive -X 1000 --no-mixed -no-discordant ${ }^{81}$. Optical and PCR duplicates were removed using Picard Tools function MarkDuplicates REMOVE_DUPLICATES $=$ true. hMeDIP-seq signal enrichment over ATAC-seq peaks was plotted using deepTools 2 computeMatrix reference-point and plotHeatmap functions.

\section{ATAC-seq data analysis}

Sea urchin ATAC-seq data was generated as part of this study, whereas lancelet and zebrafish ATAC-seq data were downloaded from GSE106428. Sequenced ATAC-seq reads in FASTQ format were trimmed using Trimmomatic software (ILLUMINACLIP:TruSeq3-PE.fa:2:30:10 SLIDINGWINDOW:5:20

LEADING:3 TRAILING:3 MINLEN:25). Properly paired 
trimmed read pairs were mapped to Spur_v3.1, B171nemr and danRer10 genome references for sea urchin, lancelet, and zebrafish, respectively, using bowtie2 v2.1.0 with the following parameters: bowtie2 -p 10 -N 1 --very-sensitive -X 2000 --nomixed --no-discordant ${ }^{81}$. Mapped reads in SAM format were converted to BAM and indexed using SAMtools ${ }^{77}$. Optical and PCR duplicates were removed using Picard Tools function MarkDuplicates REMOVE_DUPLICATES $=$ true. ATAC-seq fragment length distribution was plotted using deepTools $2^{82}$ bamPEFragmentSize --histogra. Removal of mono-, di- and trinucleosome-bound DNA fragments (and retention of the nucleosomefree fragments) and read adjustment $+4 \mathrm{bp}-5 \mathrm{bp}$ for positive and negative strand, respectively, was performed using deepTools2 alignmentSieve -ATACshift --maxFragmentLength 100. Peaks were called using MACS2 $2^{83}$ with the following parameters: macs 2 callpeak - $t$ \$input_bam $-f$ BAMPE -g \$\{genome_size\} --nomodel --nolambda -outdir \$\{OUTPUT_DIR\}-n \$name --call-summits $B-q 0.05$.

\section{RNA-seq data analysis}

Sea urchin RNA-seq data was downloaded from PRJNA81157, whereas lancelet and zebrafish RNAseq data were downloaded from GSE106430 / PRJNA416866. Illumina adapter trimming was performed using TrimGalore v0.4.0 (https://github.com/FelixKrueger/TrimGalore). Trimmed sequence reads were aligned to the Spur_v3.1, B171nemr and danRer10 reference genomes for sea urchin, lancelet and zebrafish, respectively, using STAR v2.4.0d ${ }^{84}$. Quantification of transcript abundance was performed using RSEM v1.2.2 ${ }^{85}$. TET genes analysed in this study correspond to the following IDs: WHL22.387614 (SPU_027517) for sea urchin, BL00006 for the European lancelet, and ENSDARG00000075230,ENSDARG00000076928, ENSDARG00000062646 for zebrafish.

\section{TET protein sequence and structure conservation analysis}

Sea urchin TET protein sequence was retrieved from The Universal Protein Resource (UniProt): UniProtKB - A0A7M7NF23 Methylcytosine dioxygenase TET (A0A7M7NF23_STRPU). Florida lancelet TET protein sequence was retrieved from https://ftp.ncbi.nlm.nih.gov/genomes/all/annotation
_releases/7739/100/GCF_000003815.2_Bfl_VNyy K/GCF_000003815.2_Bfl_VNyyK_protein.faa.gz, XP_035668917.1 methylcytosine dioxygenase TET1-like isoform X1 [Branchiostoma floridae]. Fruit fly, zebrafish, mouse, and human TET protein sequences were retrieved from UniProt. To predict TET protein domain structure, amino acid sequences of target sTET, bTET and zebrafish TET1, TET2, TET3 proteins in FASTA format were used as input for HMMER (http://hmmer.org/) and SWISSMODEL ${ }^{86}$. Multiple alignment of TET proteins amino acid sequences was performed using UniProt. The MSA comparison was visualised using Jalview 2.11.1.4 ${ }^{87}$. SWISS-MODEL was used to perform protein structure homology modelling ${ }^{86}$. First, amino acid sequences of target sTET, bTET and zebrafish TET1, TET2, TET3 proteins in FASTA format were used as input. 5d9y.1.A (crystal structure of human TET2-5fC complex) and 5exh.1.C (crystal structure of mTET3-CxxC domain in complex with 5carboxylcytosine DNA) were identified as the top ranked templates based on GMQE (Global Model Quality Estimate), which provides a quality estimate combining the properties from the target-template alignment and the template structure. Sequence identity between the input and 5d9y.1.A template was $57 \%$ and $64 \%$ for sea urchin sTET and lancelet sTET, respectively; and $71 \%, 75 \%, 67 \%$ for zebrafish TET1, TET2, and TET3, respectively. $5 \mathrm{~d} 9 \mathrm{y} .1$.A template was used to build a 3D model of the TET N-terminal catalytic domain. Model quality was assessed by QMEANDisCo global (the entire structure) quality score, which is a quality measurement ranging between 0 to 1 ; the closer the score to 1, the higher the expected quality. QMEANDisCo global score was 0.72 and 0.70 for sea urchin sTET and lancelet sTET, respectively; and $0.62,0.57$ and 0.80 for zebrafish TET1, TET2, and TET3, respectively.

\section{Analysis of developmentally hydroxymethylated zebrafish gene orthologs}

To assess the temporal and spatial expression of zebrafish orthologs of sea urchin genes linked to $5 \mathrm{hmC}$ enrichment (ATAC hmC+ genes), we first identified orthologous genes by converting sea urchin and zebrafish gene IDs to Drosophila melanogaster gene IDs using ENSEMBL BioMART tool. Next, sea urchin genes harbouring ATAC $\mathrm{hmC}+$ peaks within their promoters or gene bodies 
were identified. Zebrafish genes, orthologous to sea urchin ATAC hmC+ genes, were used in the downstream analysis. To compare developmental expression dynamics of zebrafish genes possessing distinct regulatory landscapes after three rounds of whole genome duplication, we identified zebrafish 2R/3R-ohnologues among zebrafish genes, orthologous to the sea urchin ATAC hmC+ genes. 2R and 3R ohnologues (Intermediate: $q$-score (outgroups) $<0.01$ AND $q$-score (self-comparison) $<$ 0.01) were downloaded from http://ohnologs.curie. $\mathrm{fr}^{88}$. Ohnologues were first split into three groups: (i) genes associated with 5hmC-marked ATAC-seq peaks overlapping phyloDMRs (5hmC/phylo-DMR), (ii) 5hmC-marked ATAC-seq peaks not overlapping phylo-DMRs (5hmC/no phylo-DMR) and (iii) non-5hmC ATACseq peaks not overlapping phylo-DMRs (no $5 \mathrm{hmC} /$ no phylo-DMR) in their distal regulatory domains. Zebrafish distal regulatory domains were defined using GREAT gene regulatory domain definition ${ }^{60}$. Briefly, basal regulatory domains were defined as $5 \mathrm{~kb}$ upstream and $1 \mathrm{~kb}$ downstream of the TSS, regardless of the neighbouring genes. To define distal regulatory domains, basal regulatory domains of each gene were extended upstream and downstream to the neighbouring gene's basal domain but no more than $1000 \mathrm{~kb}$ in each direction. PhyloDMRs were downloaded from ${ }^{19}$. 24hpf and $48 \mathrm{hpf}$ ATAC-seq peaks were downloaded from DANIOCODE (ATAC-seq_Skarmeta_Lab_0001AS, https://danio-code.zfin.org) ${ }^{19,89}$. Only the ohnologues with at least two different regulatory landscapes (5hmC/phylo-DMR, 5hmC/no phylo-DMR or no $5 \mathrm{hmC} / \mathrm{no}$ phylo-DMR) were considered in the downstream bulk RNA-seq and scRNA-seq analysis.

\section{scRNA-seq data analysis}

Zebrafish 24hpf embryo single cell RNA-seq InDrop data was downloaded from GSE112294 ${ }^{66}$. The data was converted to a file format that resembles $10 \mathrm{X}$ Chromium CellRanger output using the indRop $\mathrm{R}$ package (https://github.com/caleblareau/indRop) and processed using the standard Seurat pipeline with the parameters suggested by developers ${ }^{90}$. Briefly, single cells with less than 200 detected genes, more than 4000 detected genes, indicative of empty droplets and duplicate cells in the droplet, respectively, were discarded. Cells with total UMI (transcript) counts less than 1000 and more than
15,000 were excluded from the analysis. Read counts were normalized by a factor of 10,000 and $\log$ transformed. Highly variable features were identified using FindVariableFeatures function. The number of UMIs per cell was regressed out using Seurat's ScaleData function. RunPCA was used to perform linear dimensionality reduction on scaled data using determined highly variable features. Cell clustering was performed using FindNeighbors(object, reduction $=$ "pca", dims $=1: 20)$ and FindClusters (object, resolution $=0.5$, algorithm $=$ 1). Non-linear dimensionality reduction was performed using Uniform Manifold Approximation and Projection: RunUMAP(object, reduction = "pca", dims = 1:20).

\section{scRNA-seq downstream analysis}

FindAllMarkers function was used to define gene markers for every cluster in the $24 \mathrm{hpf}$ zebrafish embryo. Top 20 genes identified as cluster features ${ }^{66}$ were matched with the obtained gene markers and used to assign cell clusters to different tissue types. AddModuleScore function was used to define module scores for a set of genes harbouring a $5 \mathrm{hmC}$ peak and a phyloDMR (5hmC / phyloDMR), and genes harbouring a $5 \mathrm{hmC}$ peak not overlapping a phyloDMR (5hmC / no phyloDMR), and genes without a $5 \mathrm{hmC}$ peak and without a phyloDMR (no 5hmC / no phyloDMR). Positive module score indicates that a given module of genes is more highly expressed in a certain cell than what would be expected from the average expression of this gene module across all cells in the population. Module score was then $\mathrm{z}$-transformed and used in the FeaturePlot. To generate 'upset' plots, PrctCellExpringGene function was used to calculate the percentage of cells expressing each of the genes in the $5 \mathrm{hmC} /$ phyloDMR, $5 \mathrm{hmC} /$ no phyloDMR and no $5 \mathrm{hmC} / \mathrm{no}$ phyloDMR gene sets. Genes with nonzero expression counts in more than $25 \%$ of cells in any given cluster, were deemed expressed in that cluster. UpSetR package upset function was used to display the number of genes in the gene set expressed per cluster, as well as the number of genes expressed across different numbers of clusters.

\section{DNA sequence motif analysis}

Motif analysis of the $5 \mathrm{hmC}$-marked ATAC-seq regions (+/- 250 bp from the ATAC-seq peak summit) associated with promoters or gene bodies 
was performed using HOMER findMotifsGenome.pl using the default HOMER background normalised for GC content ${ }^{91}$. Motifs were visualised using $\mathrm{R}$ package ggseqlogo $^{92}$.

\section{Data availability}

MethylC-Seq, ACE-seq and ATAC-seq data generated in this study are available from NCBI Gene Expression Omnibus ${ }^{93}$ (GEO SuperSeries accession number GSE188334). Specifically, sea urchin and lancelet MethylC-seq are available under GSE188333. Sea Urchin, lancelet and zebrafish ACE-seq data are available under GSE188331. Sea Urchin, lancelet and zebrafish hMeDIP-seq data are available under GSE188332. Sea urchin ATAC-seq data are available under GSE186363. Public lancelet and zebrafish ATAC-seq data are available under GSE106428. Public sea urchin RNA-seq data are available under PRJNA81157. Public lancelet and zebrafish RNA-seq data are available under GSE106430 / PRJNA416866. Public single cell RNA-seq data of zebrafish $24 \mathrm{hpf}$ embryo is available under GSE112294.

\section{Acknowledgements}

The authors would like to thank Alex de Mendoza for critical reading of the manuscript, Patrick Leahy for providing adult sea urchins, and Davide Caramiello for sea urchin care. Australian Research Council (ARC) Discovery Project [DP190103852] to OB supported this work. Drawings of adult sea urchin, lancelet, and zebrafish were created with BioRender.com.

\section{Competing interests}

The authors declare no competing interests.

\section{Author contributions}

OB and KS designed the study. MIA, HE and JLGS contributed to concept and study design. Sea urchin embryo and adult tissue collection and DNA extraction were performed by DV and MIA. Lancelet embryo and adult tissue collection and DNA extraction were performed by SB and HE. MethylCseq library preparation and sequencing was carried out by PED and SER. ACE-seq library preparation and sequencing was carried out by KS. Sea urchin ATAC-seq library preparation was carried out by MSM, DV, MIA and IM and sequencing was carried out by MSM and IM. KS performed data analysis. $\mathrm{OB}$ and RJW participated in data analysis. KS and OB wrote the manuscript. All authors discussed the results and commented on the manuscript.

\section{References}

1 Davidson, E. H. et al. A genomic regulatory network for development. Science 295, 1669-1678, doi:10.1126/science.1069883 (2002).

2 Woolfe, A. et al. Highly conserved non-coding sequences are associated with vertebrate development. PLoS Biol 3, e7, doi:10.1371/journal.pbio.0030007 (2005).

3 Bogdanovic, O. et al. Dynamics of enhancer chromatin signatures mark the transition from pluripotency to cell specification during embryogenesis. Genome Res 22, 2043-2053, doi:10.1101/gr.134833.111 (2012).

4 Schwaiger, M. et al. Evolutionary conservation of the eumetazoan gene regulatory landscape. Genome Res 24, 639-650, doi:10.1101/gr.162529.113 (2014).

5 Schubeler, D. Function and information content of DNA methylation. Nature 517, 321-326, doi:10.1038/nature14192 (2015).

6 Lee, H. J., Hore, T. A. \& Reik, W. Reprogramming the methylome: erasing memory and creating diversity. Cell Stem Cell 14, 710-719, doi:10.1016/j.stem.2014.05.008 (2014).

7 Greenberg, M. V. C. Get Out and Stay Out: New Insights Into DNA Methylation Reprogramming in Mammals. Front Cell Dev Biol 8, 629068, doi:10.3389/fcell.2020.629068 (2020).

8 Rougier, N. et al. Chromosome methylation patterns during mammalian preimplantation development. Genes Dev 12, 2108-2113 (1998).

9 Ratnam, S. et al. Dynamics of Dnmt1 methyltransferase expression and intracellular localization during oogenesis and preimplantation development. Dev Biol 245, 304-314, doi:10.1006/dbio.2002.0628 (2002). 
10 Cardoso, M. C. \& Leonhardt, H. DNA methyltransferase is actively retained in the cytoplasm during early development. J Cell Biol 147, 25-32 (1999).

11 Mulholland, C. B. et al. Recent evolution of a TET-controlled and DPPA3/STELLA-driven pathway of passive DNA demethylation in mammals. Nat Commun 11, 5972, doi:10.1038/s41467-020-196031 (2020).

12 Nakamura, T. et al. PGC7/Stella protects against DNA demethylation in early embryogenesis. Nat Cell Biol 9, 64-71, doi:10.1038/ncb1519 (2007).

13 Wossidlo, M. et al. 5-Hydroxymethylcytosine in the mammalian zygote is linked with epigenetic reprogramming. Nat Commun 2, 241, doi:10.1038/ncomms 1240 (2011).

$14 \mathrm{Gu}, \mathrm{T}$. P. et al. The role of Tet3 DNA dioxygenase in epigenetic reprogramming by oocytes. Nature 477, 606-610, doi:10.1038/nature10443 (2011).

15 Amouroux, R. et al. De novo DNA methylation drives 5hmC accumulation in mouse zygotes. Nat Cell Biol 18, 225-233, doi:10.1038/ncb3296 (2016).

16 Ito, S. et al. Tet proteins can convert 5-methylcytosine to 5-formylcytosine and 5-carboxylcytosine. Science 333, 1300-1303, doi:10.1126/science.1210597 (2011).

17 Kohli, R. M. \& Zhang, Y. TET enzymes, TDG and the dynamics of DNA demethylation. Nature 502, 472-479, doi:10.1038/nature12750 (2013).

18 Dai, H. Q. et al. TET-mediated DNA demethylation controls gastrulation by regulating Lefty-Nodal signalling. Nature 538, 528-532, doi:10.1038/nature20095 (2016).

19 Bogdanovic, O. et al. Active DNA demethylation at enhancers during the vertebrate phylotypic period. Nat Genet 48, 417-426, doi:10.1038/ng.3522 (2016).

20 Ross, S. E. \& Bogdanovic, O. Generation and Molecular Characterization of Transient tet1/2/3 Zebrafish Knockouts. Methods Mol Biol 2272, 281-318, doi:10.1007/978-1-0716-1294-1_17 (2021). Hon, G. C. et al. $5 \mathrm{mC}$ oxidation by Tet 2 modulates enhancer activity and timing of transcriptome reprogramming during differentiation. Mol Cell 56, 286-297, doi:10.1016/j.molcel.2014.08.026 (2014).

Li, C. et al. Overlapping Requirements for Tet2 and Tet3 in Normal Development and Hematopoietic Stem Cell Emergence. Cell Rep 12, 1133-1143, doi:10.1016/j.celrep.2015.07.025 (2015).

23 Koh, K. P. et al. Tet1 and Tet2 regulate 5-hydroxymethylcytosine production and cell lineage specification in mouse embryonic stem cells. Cell Stem Cell 8, 200-213, doi:10.1016/j.stem.2011.01.008 (2011).

$24 \mathrm{Li}, \mathrm{X}$. et al. Tet proteins influence the balance between neuroectodermal and mesodermal fate choice by inhibiting Wnt signaling. Proc Natl Acad Sci U S A 113, E8267-E8276, doi:10.1073/pnas.1617802113 (2016).

Blattler, A. et al. Global loss of DNA methylation uncovers intronic enhancers in genes showing expression changes. Genome Biol 15, 469, doi:10.1186/s13059-014-0469-0 (2014).

Suzuki, M. M. \& Bird, A. DNA methylation landscapes: provocative insights from epigenomics. Nat Rev Genet 9, 465-476, doi:10.1038/nrg2341 (2008).

27 Bewick, A. J., Vogel, K. J., Moore, A. J. \& Schmitz, R. J. Evolution of DNA Methylation across Insects. Mol Biol Evol 34, 654-665, doi:10.1093/molbev/msw264 (2017).

28 de Mendoza, A., Lister, R. \& Bogdanovic, O. Evolution of DNA Methylome Diversity in Eukaryotes. J Mol Biol, doi:10.1016/j.jmb.2019.11.003 (2019).

Rae, P. M. \& Steele, R. E. Absence of cytosine methylation at C-C-G-G and G-C-G-C sites in the rDNA coding regions and intervening sequences of Drosophila and the rDNA of other insects. Nucleic Acids Res 6, 2987-2995, doi:10.1093/nar/6.9.2987 (1979).

30 Simpson, V. J., Johnson, T. E. \& Hammen, R. F. Caenorhabditis elegans DNA does not contain 5methylcytosine at any time during development or aging. Nucleic Acids Res 14, 6711-6719 (1986). de Mendoza, A. et al. Convergent evolution of a vertebrate-like methylome in a marine sponge. Nat Ecol Evol 3, 1464-1473, doi:10.1038/s41559-019-0983-2 (2019). 
Neri, F. et al. Intragenic DNA methylation prevents spurious transcription initiation. Nature $\mathbf{5 4 3}, \mathbf{7 2 -}$ 77, doi:10.1038/nature21373 (2017).

33 Parker, M. J., Weigele, P. R. \& Saleh, L. Insights into the Biochemistry, Evolution, and Biotechnological Applications of the Ten-Eleven Translocation (TET) Enzymes. Biochemistry 58, 450-467, doi:10.1021/acs.biochem.8b01185 (2019).

34 Delatte, B. et al. RNA biochemistry. Transcriptome-wide distribution and function of RNA hydroxymethylcytosine. Science 351, 282-285, doi:10.1126/science.aac5253 (2016).

Wojciechowski, M. et al. Insights into DNA hydroxymethylation in the honeybee from in-depth analyses of TET dioxygenase. Open Biol 4, doi:10.1098/rsob.140110 (2014).

36 Cingolani, P. et al. Intronic non-CG DNA hydroxymethylation and alternative mRNA splicing in honey bees. BMC Genomics 14, 666, doi:10.1186/1471-2164-14-666 (2013).

37 Moroz, L. L. et al. The ctenophore genome and the evolutionary origins of neural systems. Nature 510, 109-114, doi:10.1038/nature13400 (2014).

38 Marletaz, F. et al. Amphioxus functional genomics and the origins of vertebrate gene regulation. Nature, doi:10.1038/s41586-018-0734-6 (2018).

39 Sea Urchin Genome Sequencing, C. et al. The genome of the sea urchin Strongylocentrotus purpuratus. Science 314, 941-952, doi:10.1126/science.1133609 (2006).

40 Urich, M. A., Nery, J. R., Lister, R., Schmitz, R. J. \& Ecker, J. R. MethylC-seq library preparation for base-resolution whole-genome bisulfite sequencing. Nat Protoc 10, 475-483, doi:10.1038/nprot.2014.114 (2015).

41 Schutsky, E. K. et al. Nondestructive, base-resolution sequencing of 5-hydroxymethylcytosine using a DNA deaminase. Nat Biotechnol, doi:10.1038/nbt.4204 (2018).

Putnam, N. H. et al. The amphioxus genome and the evolution of the chordate karyotype. Nature 453, 1064-1071, doi:10.1038/nature06967 (2008).

$\mathrm{Xu}, \mathrm{Y}$. et al. Tet3 CXXC domain and dioxygenase activity cooperatively regulate key genes for Xenopus eye and neural development. Cell 151, 1200-1213, doi:10.1016/j.cell.2012.11.014 (2012). Jessop, P., Ruzov, A. \& Gering, M. Developmental Functions of the Dynamic DNA Methylome and Hydroxymethylome in the Mouse and Zebrafish: Similarities and Differences. Front Cell Dev Biol 6, 27, doi:10.3389/fcell.2018.00027 (2018).

45 Holland, P. W., Garcia-Fernandez, J., Williams, N. A. \& Sidow, A. Gene duplications and the origins of vertebrate development. Dev Suppl, 125-133 (1994).

Rasmussen, K. D. \& Helin, K. Role of TET enzymes in DNA methylation, development, and cancer. Genes Dev 30, 733-750, doi:10.1101/gad.276568.115 (2016).

47 Long, H. K., Blackledge, N. P. \& Klose, R. J. ZF-CxxC domain-containing proteins, CpG islands and the chromatin connection. Biochem Soc Trans 41, 727-740, doi:10.1042/BST20130028 (2013). Zhang, W. et al. Isoform Switch of TET1 Regulates DNA Demethylation and Mouse Development. Mol Cell 64, 1062-1073, doi:10.1016/j.molcel.2016.10.030 (2016).

49 Tu, Q., Cameron, R. A., Worley, K. C., Gibbs, R. A. \& Davidson, E. H. Gene structure in the sea urchin Strongylocentrotus purpuratus based on transcriptome analysis. Genome Res 22, 2079-2087, doi:10.1101/gr.139170.112 (2012).

50 Bertrand, S. et al. The Ontology of the Amphioxus Anatomy and Life Cycle (AMPHX). Front Cell Dev Biol 9, 668025, doi:10.3389/fcell.2021.668025 (2021).

51 Carvalho, J. E. et al. An Updated Staging System for Cephalochordate Development: One Table Suits Them All. Front Cell Dev Biol 9, 668006, doi:10.3389/fcell.2021.668006 (2021).

$\mathrm{Xu}, \mathrm{X}$. et al. Evolutionary transition between invertebrates and vertebrates via methylation reprogramming in embryogenesis. Natl Sci Rev 6, 993-1003, doi:10.1093/nsr/nwz064 (2019). Hon, G. C. et al. Epigenetic memory at embryonic enhancers identified in DNA methylation maps from adult mouse tissues. Nat Genet 45, 1198-1206, doi:10.1038/ng.2746 (2013).

$54 \mathrm{Yu}$, M. et al. Base-resolution analysis of 5-hydroxymethylcytosine in the mammalian genome. Cell 149, 1368-1380, doi:10.1016/j.cell.2012.04.027 (2012). 
Skvortsova, K. et al. Comprehensive evaluation of genome-wide 5-hydroxymethylcytosine profiling approaches in human DNA. Epigenetics Chromatin 10, 16, doi:10.1186/s13072-017-0123-7 (2017). Kamstra, J. H., Loken, M., Alestrom, P. \& Legler, J. Dynamics of DNA hydroxymethylation in zebrafish. Zebrafish 12, 230-237, doi:10.1089/zeb.2014.1033 (2015).

Seritrakul, P. \& Gross, J. M. Tet-mediated DNA hydroxymethylation regulates retinal neurogenesis by modulating cell-extrinsic signaling pathways. PLoS Genet 13, e1006987, doi:10.1371/journal.pgen.1006987 (2017).

Skvortsova, K. \& Bogdanovic, O. TAB-seq and ACE-seq Data Processing for Genome-Wide DNA hydroxymethylation Profiling. Methods Mol Biol 2272, 163-178, doi:10.1007/978-1-0716-1294-1_9 (2021).

59 Reimand, J., Kull, M., Peterson, H., Hansen, J. \& Vilo, J. g:Profiler--a web-based toolset for functional profiling of gene lists from large-scale experiments. Nucleic Acids Res 35, W193-200, doi:10.1093/nar/gkm226 (2007).

60 McLean, C. Y. et al. GREAT improves functional interpretation of cis-regulatory regions. Nat Biotechnol 28, 495-501, doi:10.1038/nbt.1630 (2010).

61 Zeitlinger, J. \& Stark, A. Developmental gene regulation in the era of genomics. Dev Biol 339, 230239, doi:10.1016/j.ydbio.2009.12.039 (2010).

62 Vanzan, L. et al. High throughput screening identifies SOX2 as a super pioneer factor that inhibits DNA methylation maintenance at its binding sites. Nat Commun 12, 3337, doi:10.1038/s41467-02123630-x (2021).

63 Yang, Y. A. et al. FOXA1 potentiates lineage-specific enhancer activation through modulating TET1 expression and function. Nucleic Acids Res 44, 8153-8164, doi:10.1093/nar/gkw498 (2016). Ficz, G. et al. Dynamic regulation of 5-hydroxymethylcytosine in mouse ES cells and during differentiation. Nature 473, 398-402, doi:10.1038/nature10008 (2011).

Pastor, W. A. et al. Genome-wide mapping of 5-hydroxymethylcytosine in embryonic stem cells. Nature 473, 394-397, doi:10.1038/nature10102 (2011).

66 Wagner, D. E. et al. Single-cell mapping of gene expression landscapes and lineage in the zebrafish embryo. Science 360, 981-987, doi:10.1126/science.aar4362 (2018).

67 Yang, H. et al. A map of cis-regulatory elements and 3D genome structures in zebrafish. Nature $\mathbf{5 8 8}$, 337-343, doi:10.1038/s41586-020-2962-9 (2020).

Deaton, A. M. \& Bird, A. CpG islands and the regulation of transcription. Genes Dev 25, 1010-1022, doi:10.1101/gad.2037511 (2011).

Hore, T. A. et al. Retinol and ascorbate drive erasure of epigenetic memory and enhance reprogramming to naive pluripotency by complementary mechanisms. Proc Natl Acad Sci U S A 113, 12202-12207, doi:10.1073/pnas.1608679113 (2016).

70 Pastor, W. A., Aravind, L. \& Rao, A. TETonic shift: biological roles of TET proteins in DNA demethylation and transcription. Nat Rev Mol Cell Biol 14, 341-356, doi:10.1038/nrm3589 (2013). Levin, M. et al. The mid-developmental transition and the evolution of animal body plans. Nature 531, 637-641, doi:10.1038/nature16994 (2016).

72 Fuentes, M. et al. Insights into spawning behavior and development of the European amphioxus (Branchiostoma lanceolatum). J Exp Zool B Mol Dev Evol 308, 484-493, doi:10.1002/jez.b.21179 (2007).

73 Wang, T. et al. Bisulfite-Free Sequencing of 5-Hydroxymethylcytosine with APOBEC-Coupled Epigenetic Sequencing (ACE-Seq). Methods Mol Biol 2198, 349-367, doi:10.1007/978-1-0716-08760_27 (2021).

Buenrostro, J. D., Giresi, P. G., Zaba, L. C., Chang, H. Y. \& Greenleaf, W. J. Transposition of native chromatin for fast and sensitive epigenomic profiling of open chromatin, DNA-binding proteins and nucleosome position. Nat Methods 10, 1213-1218, doi:10.1038/nmeth.2688 (2013). Embryos. Methods Mol Biol 2219, 253-265, doi:10.1007/978-1-0716-0974-3_16 (2021). 
Chen, H., Smith, A. D. \& Chen, T. WALT: fast and accurate read mapping for bisulfite sequencing. Bioinformatics 32, 3507-3509, doi:10.1093/bioinformatics/btw490 (2016).

77 Li, H. et al. The Sequence Alignment/Map format and SAMtools. Bioinformatics 25, 2078-2079, doi:10.1093/bioinformatics/btp352 (2009).

Bolger, A. M., Lohse, M. \& Usadel, B. Trimmomatic: a flexible trimmer for Illumina sequence data. Bioinformatics 30, 2114-2120, doi:10.1093/bioinformatics/btu170 (2014).

Park, Y. \& Wu, H. Differential methylation analysis for BS-seq data under general experimental design. Bioinformatics 32, 1446-1453, doi:10.1093/bioinformatics/btw026 (2016).

80 Buske, F. A., French, H. J., Smith, M. A., Clark, S. J. \& Bauer, D. C. NGSANE: a lightweight production informatics framework for high-throughput data analysis. Bioinformatics 30, 1471-1472, doi:10.1093/bioinformatics/btu036 (2014).

81 Langmead, B. \& Salzberg, S. L. Fast gapped-read alignment with Bowtie 2. Nat Methods 9, 357-359, doi:10.1038/nmeth.1923 (2012).

82 Ramirez, F. et al. deepTools2: a next generation web server for deep-sequencing data analysis. Nucleic Acids Res 44, W160-165, doi:10.1093/nar/gkw257 (2016).

83 Zhang, Y. et al. Model-based analysis of ChIP-Seq (MACS). Genome Biol 9, R137, doi:10.1186/gb2008-9-9-r137 (2008).

84 Dobin, A. et al. STAR: ultrafast universal RNA-seq aligner. Bioinformatics 29, 15-21, doi:10.1093/bioinformatics/bts635 (2013).

85 Li, B. \& Dewey, C. N. RSEM: accurate transcript quantification from RNA-Seq data with or without a reference genome. BMC Bioinformatics 12, 323, doi:10.1186/1471-2105-12-323 (2011).

Waterhouse, A. et al. SWISS-MODEL: homology modelling of protein structures and complexes. Nucleic Acids Res 46, W296-W303, doi:10.1093/nar/gky427 (2018).

87 Waterhouse, A. M., Procter, J. B., Martin, D. M., Clamp, M. \& Barton, G. J. Jalview Version 2--a multiple sequence alignment editor and analysis workbench. Bioinformatics 25, 1189-1191, doi:10.1093/bioinformatics/btp033 (2009).

Singh, P. P. \& Isambert, H. OHNOLOGS v2: a comprehensive resource for the genes retained from whole genome duplication in vertebrates. Nucleic Acids Res 48, D724-D730, doi:10.1093/nar/gkz909 (2020).

89 Baranasic, D. et al. (bioRxiv, 2021).

90 Satija, R., Farrell, J. A., Gennert, D., Schier, A. F. \& Regev, A. Spatial reconstruction of single-cell gene expression data. Nat Biotechnol 33, 495-502, doi:10.1038/nbt.3192 (2015).

91 Heinz, S. et al. Simple combinations of lineage-determining transcription factors prime cis-regulatory elements required for macrophage and B cell identities. Mol Cell 38, 576-589, doi:10.1016/j.molcel.2010.05.004 (2010). Wagih, O. ggseqlogo: a versatile R package for drawing sequence logos. Bioinformatics 33, 36453647, doi:10.1093/bioinformatics/btx469 (2017).

93 Edgar, R., Domrachev, M. \& Lash, A. E. Gene Expression Omnibus: NCBI gene expression and hybridization array data repository. Nucleic Acids Res 30, 207-210 (2002). 